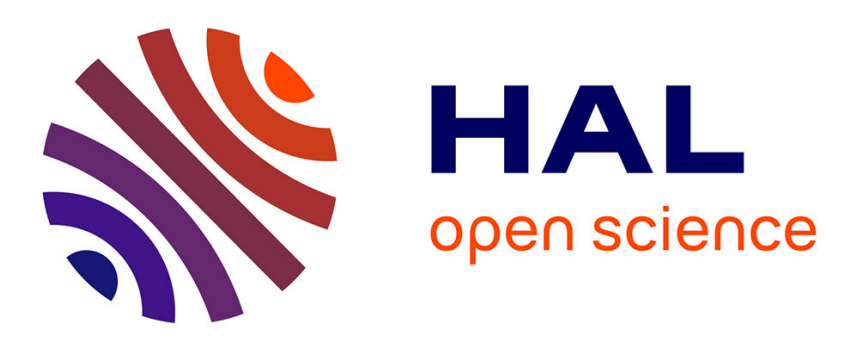

\title{
Dynamic behaviour of elastic metamaterials containing soft elliptic fibers
}

\author{
Guy Bonnet, V. Monchiet
}

\section{To cite this version:}

Guy Bonnet, V. Monchiet. Dynamic behaviour of elastic metamaterials containing soft elliptic fibers. Journal of the Mechanics and Physics of Solids, 2020, 140, pp.103953. 10.1016/j.jmps.2020.103953 . hal-02529182

\section{HAL Id: hal-02529182 \\ https://hal.science/hal-02529182}

Submitted on 2 Apr 2020

HAL is a multi-disciplinary open access archive for the deposit and dissemination of scientific research documents, whether they are published or not. The documents may come from teaching and research institutions in France or abroad, or from public or private research centers.
L'archive ouverte pluridisciplinaire HAL, est destinée au dépôt et à la diffusion de documents scientifiques de niveau recherche, publiés ou non, émanant des établissements d'enseignement et de recherche français ou étrangers, des laboratoires publics ou privés. 


\title{
Dynamic behaviour of elastic metamaterials containing soft elliptic fibers
}

\author{
G. Bonnet ${ }^{\mathrm{a}, *}$, V. Monchiet $^{\mathrm{a}}$ \\ ${ }^{a}$ Université Paris-Est,Laboratoire Modélisation et Simulation Multi Echelle, MSME UMR \\ 8208 CNRS, 5 bd Descartes, 77454 Marne-la-Vallée, France
}

\begin{abstract}
The dynamic behaviour of composites containing long soft fibers of elliptic cross section is studied in the frequency range where the material behaves as a metamaterial. The results of homogenization theory for composites containing soft inclusions are recalled, showing the conditions that lead to a frequency dependent dynamic density coming from the inner resonance of soft inclusions. The dynamic density is expressed from the resonance frequencies and participation factors of the inner inclusions obtained by solving a localization problem. The solution to this problem is obtained for a composite containing long fibers of elliptic cross section by using an expansion into Mathieu functions. The set of resonance frequencies and participation factors can be split into different subsets corresponding to the different symmetries of localization problems related to different directions of the macroscopic acceleration. All results are found to be consistent with the extreme cases of aspect ratios corresponding to stratified media (plane inclusions) and circular fibers.

Keywords: Metamaterials, Elasticity, Resonance, Dynamic density, Acoustic polarizer
\end{abstract}

\footnotetext{
* Corresponding author

Email address: guy.bonnet@u-pem.fr (G. Bonnet)

$U R L$ : www.msme.u-pem.fr (G. Bonnet)
} 


\section{Introduction}

Numerous works were performed during the last decades on metamaterials, firstly in the field of electromagnetism and more recently in the field of acoustics. This field of research can be traced back to 1968 Veselago's paper (Veselago, 1968) on electromagnetism, as explained in Shamonina \& Solvmar (2007). For acoustic materials, the main aspect of the behaviour of metamaterials is the occurrence of bandgaps around given frequencies, similarly to the case of phononic crystals. So, an experimental evidence for acoustic band gaps in two-dimensional composites with fibers was obtained by Vasseur et al. (1998) in such phononic crystals. From a fundamental point of view, it appears that interesting properties of acoustic metamaterials come from resonance, either due to the collective behaviour of inclusions or to the presence of local resonators (Milton, 2007; Milton \& Willis, 2007). This last case corresponds to the occurrence of inner resonance. The resonators can be either homogeneous inclusions, composite inclusions, beams or plates. Numerous papers in this field were published that either study the properties of metamaterials from a theoretical approach (Huang \& Sun, 2011, 2012; Lee et al., 2009; Baz, 2010; Bigoni et al. 2013) or display experimentally the properties of such materials (Liu et al., 2000; Sheng et al., 2003; Naify et al., 2012; Yao et al., 2008; Park et al., 2012). An important aspect of these works is that the dynamic behaviour exhibits an "effective dynamic mass density", that becomes negative at certain frequencies, as predicted or observed by several authors (Auriault \& Bonnet, 1985; Auriault, 1994; Yao et al., 2008; Liu et al., 2000; Yang et al., 2008).

In the following, the study will be restricted to linearly elastic composites made of homogeneous inclusions. Even so, the amount of literature devoted to this field is impressive. Obtaining the dynamic effective properties of elastic composites has been effected early by extending the homogenization methods used for elastostatics, as in the paper of Willis (1981). The development of this approach led to a model often cited as "Willis model" characterized by constitutive equations allowing one to compute the macroscopic stress tensor 
and momentum from macroscopic deformation and velocity (Milton \& Willis, 2007). These constitutive equations are non-local in space in most situations, being usually built using wave-vectors, except for the limit case of vanishing wavenumbers, as in Willis (2009). The original "Willis model" was built in the context of statistical homogenization. However, this model was carefully revisited in the context of periodic media by Nassar et al. (2015). Other homogenization approaches are developed, like the one of Sridhar et al. (2018), that departs from classical approaches where the macroscopic variables are volume averages of local ones.

An usual method to study elastodynamic problems in the case of periodic media is to provide their solution for waves propagating through a periodic composite by decomposition into Bloch waves (Krokhin et al., 2003; Nemat-Nasser et al., 2011; Norris et al., 2012). This method allows one to study the collective behaviour of the periodic system while inner resonance can come from inner, independent, resonators (Zhou et al., 2012). The solutions depend essentially on the ratios of physical properties of the components of the composite (elastic parameters and mass densities). Therefore, a strong physical insight can be gained using an asymptotic series expansion of the dynamic solution for different assumptions on the ratios between physical parameters: elastic coefficients and mass densities. First results showing by such a method the occurrence of frequency bandgaps in elastic composites were obtained in early works (Auriault \& Bonnet, 1985; Auriault, 1994) but, with the development of the ideas on metamaterials, new results have been obtained more recently with the use of asymptotic expansions in dynamic elasticity (Parnell \& Abrahams, 2006; Avila et al.,, 2005; Babych et al., 2008; Auriault \& Boutin, 2012; Soubestre \& Boutin, 2012; Craster et al., 2010; Rallu et al., 2018). It is worthwhile noting that, compared with results coming from other publications in this field, an important aspect does appear in the papers written by Auriault and al. that are at the basis of our work (Auriault \& Bonnet, 1985; Auriault, 1994; Auriault \& Boutin,

60 2012): it is indeed shown in these papers that different scalings of physical properties lead to different macroscopic behaviours. As a consequence, it is of prime 
importance to characterize the scaling properties leading to a specific dynamic behaviour. As it will be seen in section 2, the homogenization method shows that a two phase matrix composite with a large contrast of elastic properties but comparable densities enters in the field of materials characterized by inner resonance which constitute a large category of metamaterials (Boutin et al., 2018; Zhou et al., 2012). In these composite materials, the inner resonance can be obtained by inner resonators made of homogeneous or composite inclusions. These materials display a dynamic density, that is characterized mainly by the resonance frequencies of the inclusions. Obviously, the behaviour that is obtained from asymptotic homogenization should be consistent with more sophisticated models. So, Nassar et al. (2016) have shown that several models coming from asymptotic solutions can be recovered from the full "Willis model". In a recent work (Comi \& Marigo, 2020), the method of homogenization described thereafter in section 2 has been used in parallel with the computation of Bloch waves to predict the frequency bandgaps and it has been shown that the estimation of bandgaps is equivalent when using both methods under the assumption of large contrast.

The computation of dynamic density and bandgaps in the case of large contrast was effected in Bonnet \& Monchiet (2017) in the case of circular fibers and spherical inclusions. In this case, the dynamic density is either isotropic (spherical inclusions) or transversally isotropic (circular fibers), the components of the dynamic density being identical in this last case for directions of acceleration in the plane orthogonal to fibers. However, it is certainly of interest to find materials that are fully orthotropic, because in this case the bandgaps can be all different between themselves for directions of motion along different axes. The aim of the paper is therefore to study a fiber matrix composite with a large contrast of elastic properties characterized by an elliptic cross section. In this case, the mass density of the composite displays a fully orthotropic behaviour.

In section 2, the results of the asymptotic expansion method in the case of materials with a large contrast of elastic properties containing homogeneous inclusions are briefly recalled to point explicitly the set of physical parameters 
leading to inner resonance.

In section 3, the inner motion of composite inclusions made of cylindrical

\section{Dynamic behaviour of elastic composites from homogenization by asymptotic expansion: basic results and discussion}

Different methods can be used in order to study the effective properties of heterogeneous materials, i.e. properties which are equivalent at the macroscopic scale to those of the heterogeneous material. This is usually possible when the microscopic scale (scale of heterogeneities in the microstructure) is small compared with the macroscopic scale, leading to a small "scaling factor". In

this case, the problem of periodic heterogeneous materials has been thoroughly studied by using asymptotic methods based on assumptions related to the order of magnitude of the different physical parameters defining the microstructure. Then, the solution of the problem at the microscopic scale can be obtained as a series expansion in powers of the "scaling factor". In this section, the solution by this method of the elastodynamic problem for the two-phase case (homogeneous inclusions) is recalled from Auriault \& Bonnet (1985) and Auriault \& Boutin (2012).

\subsection{The local equations}

Let us consider a periodic composite made of two elastic constituents, each one being comprised within a part $\Omega_{s}$ (volume $\left|\Omega_{s}\right|$ ) of the periodic (microscopic) 
cell $\Omega, s$ designing the species. In the following, only matrix inclusion composites will be studied and therefore $s=m$ for the matrix and $s=i$ for inclusions. These materials comply with the equations for the conservation of momentum which write for an harmonic motion at radial frequency $\omega$ :

$$
\operatorname{div}\left(\boldsymbol{\sigma}_{(s)}\right)+\rho_{(s)} \omega^{2} \mathbf{u}_{(s)}=0
$$

where $\rho_{(s)}$ is the mass density, $\mathbf{u}_{(s)}$ is the displacement field and $\boldsymbol{\sigma}_{(s)}$ the local stress tensor complying with the linearly elastic constitutive equations:

$$
\sigma_{i j(s)}=a_{i j k l(s)} \varepsilon_{k l(s)} .
$$

$\sigma_{i j(s)}$ and $a_{i j k l(s)}$ are the components of the stress, and elasticity tensors within the constituents, while $\varepsilon_{k l(s)}$ are the components of the linear strain tensor derived from the local displacement field $\mathbf{u}_{(s)}$.

All fields, except the displacement field, are assumed $\Omega$-periodic, i.e. periodic over the unit cell $\Omega$. Additionally, the continuity of the displacements and tractions at the interface $\Gamma$ between constituents is considered:

$$
[\boldsymbol{\sigma} \cdot \mathbf{N}]_{\Gamma}(M)=0
$$

and

$$
[\mathbf{u}]_{\Gamma}(M)=0
$$

for any point $M$ over $\Gamma$, where $\mathbf{N}$ denotes the normal to $\Gamma$ at $M$ and where the brackets denote the jump at the interface between the constituents:

$$
[\mathbf{f}]_{\Gamma}(M)=\mathbf{f}_{(m)}-\mathbf{f}_{(i)} .
$$

\subsection{Asymptotic expansion and scaling assumptions}

As it will be shown thereafter, the inner resonance appears when the physical parameters comply with a suitable scaling. It is customary to study this scaling by using the scale ratio given by:

$$
\epsilon=\frac{l}{L},
$$


where $l$ is a characteristic size of the periodic cell and $L$ is a characteristic size of the macroscopic domain, with $\epsilon<<1$. The main idea of the asymptotic expansion (Bensoussan et al., 1978) is to consider any field $v$ as expressed by a function of two position variables $\mathbf{x}$ and $\mathbf{y}$. The second one $\mathbf{y}$ defines practically the position of the cell within the macroscopic domain and the first one $\mathbf{x}$ is "amplified" by the factor $\frac{1}{\epsilon}$ in order to study what happens within the (small) periodic cell. Then, the asymptotic expansion of any field $v$ is performed along powers of $\epsilon$ by considering that $\mathbf{x}$ and $\mathbf{y}$ are "independent" variables, giving:

$$
v=v^{(0)}(\mathbf{x}, \mathbf{y})+\epsilon v^{(1)}(\mathbf{x}, \mathbf{y})+\epsilon^{2} v^{(2)}(\mathbf{x}, \mathbf{y})+\ldots
$$

At this stage, it is necessary to introduce a scaling between physical parameters in order to reach an interesting situation, from a physical point of view. In fact, the interesting situation, i.e. the one leading to inner resonance, is obtained when the inclusions are very soft compared with the matrix. Indeed, it has been proved in Auriault \& Bonnet (1985) that the interesting situation is obtained when the elasticity tensors of both phases comply with:

$$
\left\|\mathbf{a}_{(i)}\right\| /\left\|\mathbf{a}_{(m)}\right\|=O\left(\epsilon^{2}\right),
$$

where $\left\|\mathbf{a}_{(s)}\right\|$ is a norm of elasticity tensor $\mathbf{a}_{(s)}$. For the mass densities,

\subsection{Effective behaviour}

Under these conditions, by comparing the different terms of the asymptotic expansion (Auriault \& Bonnet, 1985; Auriault \& Boutin, 2012), the following results have been obtained: 
- The displacement within the matrix is given by:

$$
\mathbf{u}_{(m)}=\mathbf{u}_{(m)}^{(0)}(\mathbf{y})+\epsilon \mathbf{u}_{(m)}^{(1)}(\mathbf{x}, \mathbf{y}),
$$

where the local (x-dependent) displacement $\mathbf{u}_{(m)}^{(1)}$ is given by:

$$
\mathbf{u}_{(m)}^{(1)}(\mathbf{x}, \mathbf{y})=\boldsymbol{\xi}(\mathbf{x}): \mathbf{E}(\mathbf{y})+\hat{\mathbf{u}}_{(m)}^{(1)}(\mathbf{y})
$$

The last term is a contribution to the macroscopic displacement at the location of the cell (function only on the "slow" position variable $\mathbf{y}$ ) and the macroscopic deformation $\mathbf{E}=\boldsymbol{\epsilon}_{y}\left(\mathbf{u}_{(m)}^{(0)}\right)$ is obtained by differentiation of $\mathbf{u}_{(m)}^{(0)}$ with respect to the macroscopic position variable $\mathbf{y}$, denoted by the lower index. $\boldsymbol{\xi}(\mathbf{x})$ is the static "localization tensor" (Milton, 2002) which produces the local displacement when submitting, in the static case, the solid (containing voids in place of inclusions) to the macroscopic deformation $\mathbf{E}$. As a consequence, the effective elasticity tensor $\mathbf{a}_{e f f}$ is constant and given by the one of a composite material containing holes in place of soft inclusions. This is obviously consistent with the assumption on the ratio of elastic properties.

- The displacement within the inclusion is given by:

$$
\mathbf{u}_{(i)}^{(0)}(\mathbf{x}, \mathbf{y})=\mathbf{u}_{(m)}^{(0)}(\mathbf{y})+\mathbf{w}(\mathbf{x}, \mathbf{y}),
$$

where the relative displacement $\mathbf{w}(\mathbf{x}, \mathbf{y})$ is the solution of:

$$
\begin{aligned}
\operatorname{div}_{\mathbf{x}}\left(\mathbf{a}_{(i)}: \boldsymbol{\varepsilon}_{x}(\mathbf{w})\right) & +\rho_{(i)} \omega^{2} \mathbf{w} \\
& =-\rho_{(i)} \omega^{2} \mathbf{u}_{(m)}^{(0)}(\mathbf{y}),
\end{aligned}
$$

with the boundary condition $\mathbf{w}=0$ over the interface $\Gamma$ between matrix and inclusion (no relative displacement at the interface). In this equation, the divergence and $\varepsilon_{x}$ correspond now to the differentiation using the space variable $\mathbf{x}$. The right hand side of the equation corresponds to inertia forces coming from the acceleration of the matrix around the inclusion, 
that does not depend on the local variable, due to the large value of the wavelength within the (stiff) matrix.

The solution $\mathbf{w}$ of this problem is obviously linear in $\mathbf{u}_{(m)}^{(0)}$ and can be written:

$$
\mathbf{w}(\mathbf{x}, \mathbf{y}, \omega)=\boldsymbol{\alpha}(\mathbf{x}, \omega) \cdot \mathbf{u}_{(m)}^{(0)}(\mathbf{y}),
$$

where $\boldsymbol{\alpha}$ is a second order frequency dependent localization tensor. It is worthwhile noticing that this localization tensor is solution of a boundary value problem limited to the inclusion instead of concerning the overall periodic cell as in classical static homogenization.

- The effective dynamic equation is finally given by:

$$
\begin{aligned}
\operatorname{div}\left(\mathbf{a}_{e f f}: \mathbf{E}\right) & =-\omega^{2} \frac{1}{|\Omega|} \int_{\Omega} \rho \mathbf{u}^{(0)} d \Omega \\
& =-\omega^{2} \frac{1}{|\Omega|}\left[\left|\Omega_{m}\right| \rho_{(m)} \mathbf{u}_{(m)}^{(0)}+\rho_{(i)} \int_{\Omega_{i}} \mathbf{u}_{(i)}^{(0)} d \Omega\right] \\
& =-\omega^{2} \frac{1}{|\Omega|}\left[\left|\Omega_{m}\right| \rho_{(m)} \mathbf{u}_{(m)}^{(0)}+\rho_{(i)} \int_{\Omega_{i}}\left[\mathbf{u}_{(m)}^{(0)}+\mathbf{w}\right] d \Omega\right]
\end{aligned}
$$

Using the expression of $\mathbf{w}=\boldsymbol{\alpha} \cdot \mathbf{u}_{(\mathbf{m})}^{(\mathbf{0})}$ allows us to factorize $\mathbf{u}_{(m)}^{(0)}$, leading to

$$
\operatorname{div}\left(\mathbf{a}_{e f f}: \mathbf{E}\right)=-\omega^{2} \boldsymbol{\rho}_{e f f}(\omega) \cdot \mathbf{u}_{(m)}^{(0)},
$$

where $\rho_{\text {eff }}(\omega)$ is a second order tensor given by

$$
\boldsymbol{\rho}_{e f f}(\omega)=<\rho>\boldsymbol{\delta}+n_{(i)} \rho_{(i)}<\boldsymbol{\alpha}>_{(i)} .
$$

In equation (15), the first term appears as the divergence of $\boldsymbol{\Sigma}=\mathbf{a}_{\text {eff }} . \mathbf{E}$, i.e the divergence of the macroscopic stress tensor. The dynamic term on the right hand side of (14) shows that $-\omega^{2} \boldsymbol{\rho}_{\text {eff }}(\omega) \cdot \mathbf{u}_{(m)}^{(0)}$ corresponds to the time derivative of the overall momentum. In addition, it will be shown thereafter that, at low frequencies, the factor of the local acceleration $-\mathbf{u}^{(0)} \omega^{2}$ becomes the overall density. As a consequence, it is natural 
to consider this factor in the overall dynamic equation, $\rho_{\text {eff }}(\omega)$, as a generalization of the density of the composite, i.e. a frequency dependent "dynamic density".

In equation (16), $\boldsymbol{\delta}$ is the second order identity tensor, $n_{(s)}$ is the volume concentration of phase $s,\langle\cdot\rangle$ denotes the volume average over the periodic cell and $<\cdot\rangle_{(s)}$ denotes the volume average over domain $\Omega_{s}$.

As shown above, an important result is that the dynamic density can be obtained by studying the dynamic response of the inclusion when submitted to the acceleration of the matrix $-\omega^{2} \mathbf{u}_{(m)}^{(0)}$ with fixed boundary conditions (in the reference frame moving with the matrix). A first consequence is that the motion within the inclusion depends only on the (nearly constant) motion within the matrix around the inclusion: it means that there is no collective behaviour of the inclusions and no need to use the computational methods related to collective behaviour, like Bloch waves or multipole expansion. In addition, it can be shown that using Bloch waves expansion or the procedure described above from suitable scaling assumptions predict the same band-gaps within the material (Comi \& Marigo, 2020) in the case of large contrast. The obtained overall behaviour corresponds to the physical notion of inner resonance (or "locally resonant" materials), this kind of resonance being obtained often by using inner resonators acting as "spring-mass" systems, like composite inclusions (inner mass within a soft elastic coating acting as a spring), which constitute many metamaterials(Auriault \& Boutin, 2012; Bigoni et al., 2013; Huang \& Sun, 2011; Liu et al., 2000). Therefore, the important result at the basis of our study is that inner resonators can be also obtained using homogeneous inclusions instead of composite ones, such materials being obviously easier to build. There is in addition a strong similarity between the inner resonance obtained by composite inclusions and homogeneous inclusions: the elasticity tensor of the coating of composite inclusions and the one of the soft homogeneous inclusion must be of the same order compared to the one of the matrix in order to obtain inner resonance (Auriault \& Boutin, 2012), and similarly for 
the densities of the inclusions. Due to this local resonance and to the nature of the dynamic localization problem, the behaviour of a set of independent inclusions can be obtained by studying separately each inclusion and combining the contribution of all these separate inclusions. Considering a set of $N$ inclusions $\Omega_{q}, q=1 . . N$, the dynamic density is obtained by:

$$
\boldsymbol{\rho}_{e f f}(\omega)=<\rho>\boldsymbol{\delta}+\sum_{q} n_{q} \rho_{q}<\boldsymbol{\alpha}>_{q} .
$$

where all tensors $\langle\boldsymbol{\alpha}\rangle_{q}$ are obtained independently using the method defined

$$
\boldsymbol{\alpha}=\sum_{p} \frac{\omega^{2}}{\omega_{p}^{2}-\omega^{2}} \frac{\boldsymbol{u}^{p} \otimes<\boldsymbol{u}^{p}>_{(i)}}{<\left\|\mathbf{u}^{p}\right\|^{2}>_{(i)}}
$$

for radial eigenfrequencies $\omega_{p}$, and the localization tensor $\boldsymbol{\alpha}$ is an explicit function of eigenmodes given by: 
It follows from (19) that the dynamic density tends to infinity and becomes negative at each eigenfrequency of the inclusion. It can be seen also that $\boldsymbol{\alpha}$ tends to zero as the frequency tends to zero. As a consequence, the dynamic density tends to the static density at low frequencies. The effective dynamic density is a function of the volume average of $\boldsymbol{\alpha}$ over the inclusion domain $\Omega_{i}$, denoted by $\boldsymbol{h}=\langle\boldsymbol{\alpha}\rangle_{(i)}$. An important case corresponds to inclusions being symmetrical with respect to three orthogonal planes. In this case, tensor $\boldsymbol{h}$ is diagonal in a reference frame containing the symmetry planes. Its three diagonal components can be computed by applying successively the acceleration $\gamma$ of the matrix along the three axes,i.e. $\gamma=\mathbf{e}_{j}, j=1 . .3$.

Finally, the dynamic density is diagonal and its components are given by :

$$
\rho_{j j(e f f)}=<\rho>+n_{(i)} \rho_{(i)} h_{j j},
$$

where

$$
h_{j j}(\omega)=\sum_{p} K_{j j}^{p} \frac{\omega^{2}}{\omega_{p}^{2}-\omega^{2}} .
$$

For eigenmode $p$, the participation factor $K_{j j}^{p}$ is given by:

$$
K_{j j}^{p}=\frac{\left(<\mathbf{u}^{p}>_{(i)} \cdot \mathbf{e}_{j}\right)^{2}}{<\left\|\mathbf{u}^{p}\right\|^{2}>_{(i)}} .
$$

In these expressions, there is no sum over index $j$. Finally, the eigenfrequencies and eigenmodes must be obtained for each direction of $\mathbf{e}_{j}$ by solving the "localization problem". Next, the averages are computed to produce the participation factors.

\subsection{Remarks}

\subsubsection{Limit to the infinite set of eigenfrequencies}

The solution of the boundary value problem has been obtained by adding in (20) the contributions coming from the set of all eigenfrequencies. Considering the boundary value problem related to the dynamic equation within a bounded domain, it is known that this set is theoretically infinite. However, it must be noticed that the main assumption at the basis of this study is that the frequency 
range of the motion must lead to large wavelengths within the matrix. This is no more valid for high frequencies, as soon as the wavelength related to waves within the matrix becomes of the same order as the size of inclusions. The eigenfrequencies of interest are only those complying with the condition of large wavelength within the matrix. From a physical point of view, it means that the spectrum of frequencies contained within the excitation signal must comply with the condition of large wavelengths, i.e. to be below a high cut-off frequency $\omega_{c o}$. As a consequence it is only useful to obtain the eigenfrequencies below $\omega_{c o}$, higher resonance frequencies being not concerned.

\subsubsection{Fixed boundary condition and substructuring}

From the previous developments, it appears that the motion inside the soft inclusions is obtained from the solution of a boundary value problem with fixed boundaries. This can be linked to the work of Sridhar et al. (2016) that is interested in the production of an enriched macroscopic description of the dynamics of the composite. The authors use the well known "Craig-Bampton" substructuring method (Craig \& Bampton, 1968) that describes the dynamic displacement within inclusions by its projection onto the inner modes with a fixed boundary, as in the present solution.

\subsubsection{The question of infinite effective density at resonance}

There is finally a questionable point in the expression of the dynamic density: this one tends to infinity at eigenfrequencies, which is not physically acceptable. In fact, it is possible to show that taking into account a small damping within 250 the inclusions leads to finite values of the dynamic density (Auriault \& Boutin, 2012), without affecting significantly the resonance frequencies and the stop bands. In addition, it may be noticed that the displacement inside the inclusion tends also to infinite, simultaneously with the strain and stress fields. It means that the assumption of negligible stresses within the inclusions due to the large stiffness contrast is no more valid near the peak of resonance (when $\left\|\varepsilon_{(i)}\right\| \approx$ $\left.\epsilon^{-2}\left\|\varepsilon_{(m)}\right\|\right)$. This can explain why a non constant effective elasticity tensor was 
observed for stratified media in Willis (2009) for a ratio of elastic constants of 0.05 and not in a similar computation in Auriault \& Bonnet (1985). However, this appears for very large inner displacements and strains and does not affect the determination of the frequencies bandgaps, that is the most important result.

\section{Solution of the localization problem for a longitudinal motion}

This localization problem has been solved previously in the case of cylindrical homogeneous inclusions of circular cross section and in the case of spherical homogeneous inclusions (Bonnet \& Monchiet, 2017). We will now consider the case of cylindrical inclusions with an elliptic cross section, whose radii are $a$ and $b$ as shown in Fig:1

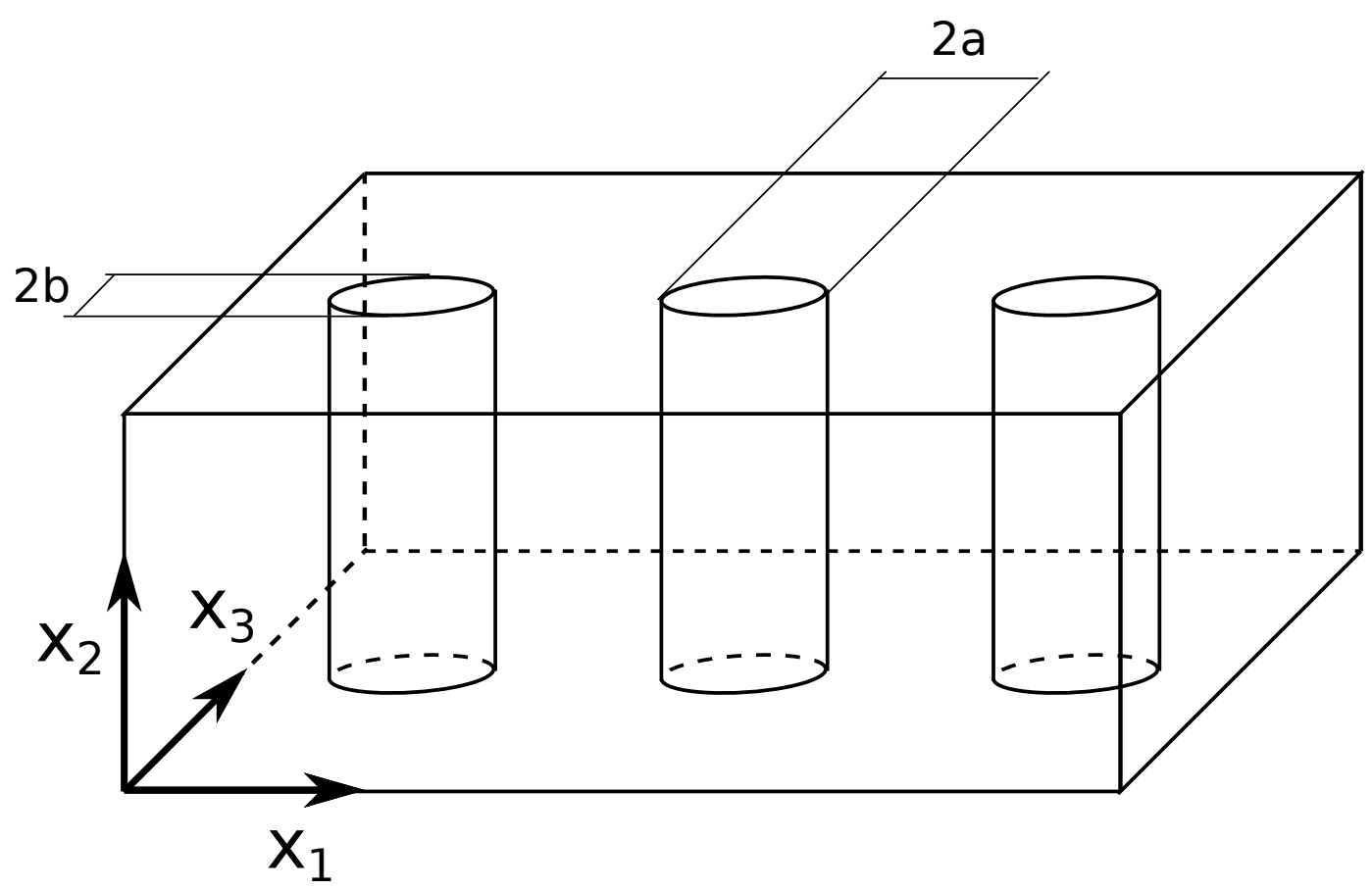

Figure 1: Geometry of the composite: fibers with elliptic cross section characterized by radii $a$ and $b$.

Different solutions can be used to obtain the solution of the problem within an elliptic domain. The solution in polar coordinates can be used for elliptic 
shapes being not too far from a circle (Bazzali, 2014; Ancey et al., 2013), but values of aspect ratios, due to the orthogonality of the harmonic solutions in elliptic coordinates (this was suggested by Hackman (1984) for ellipsoidal inclusions, but we have checked it again for elliptical inclusions during this work). In addition, it is known that the elementary solutions in elliptical coordinates, i.e. to the decoupling of the motion along Mathieu functions of different orders for a longitudinal motion, which is particularly useful. This solution in elliptical coordinates will be used in the following. As seen previously, this localization problem is solved in a reference frame moving with the matrix, the local variable being the relative displacement inside the inclusion with respect to the matrix and the excitation being provided by inertia forces $\mathbf{f}$ induced by the acceleration of the matrix around the inclusion, given by $\mathbf{f}=\rho \omega^{2} \mathbf{u}_{(m)}^{(0)}$. From a practical point of view, the symmetry of the problem induces that the tensor $\boldsymbol{h}$ is diagonal in a referential frame oriented along the symmetry axes of the fibers. Each component of this tensor can be obtained by considering successively the acceleration of the matrix around the inclusion (leading to inertia forces) directed along the natural axes of the system. There are therefore three partial localization problems. The axes are defined by the unit vectors $\mathbf{e}_{1}, \mathbf{e}_{2}, \mathbf{e}_{3} . \mathbf{e}_{3}$ is directed along the direction of the fibers and $\mathbf{e}_{1}, \mathbf{e}_{2}$ are directed along the axes of the elliptic cross section as it can be seen in Fig. 1, The main axis of the ellipse is oriented along $\mathbf{e}_{1}$. For the acceleration along $\mathbf{e}_{3}$, the motion is antiplane, while a plane motion occurs in the two other cases.

Due to the nature of the chosen accelerations and to the geometry of the fibers, it can be shown that plane and antiplane motions do not depend on $x_{3}$. In the following, the three partial localization problems will be solved successively, this section being devoted to the longitudinal motion. From now on, it is obvious that the subscripts $x$ and $y$ are no more useful in the differential operators describing the localization problems and will be suppressed. Concerning the elastic parameters, the material of the fibers will be considered as isotropic and 
homogeneous, characterized by the Lamé constants $\lambda, \mu$.

\subsection{Solution of the antiplane problem}

The antiplane motion is governed by the equation

$$
\mu \Delta w_{3}+\rho \omega^{2} w_{3}+f_{3}=0
$$

The form of this equation is the one of Helmholtz equation. Its solution is given in classical books for elliptic coordinates.

\subsubsection{Elliptic coordinates}

The elliptic coordinates are related to cartesian coordinates $x_{1}, x_{2}$ by the following relations (Morse \& Feshbach, 1953):

$$
\begin{aligned}
& x_{1}=c \cosh \xi \cos \eta \\
& x_{2}=c \sinh \xi \sin \eta,
\end{aligned}
$$

where $0 \leq \xi<\infty$ and $0 \leq \eta<2 \pi, \xi$ is the radial coordinate $(0 \leq \xi<\infty)$ and $\eta$ is the angular coordinate $(0 \leq \eta<2 \pi), 2 c$ being the focal length of the ellipse. The axes of the ellipse are $c \cosh \xi$ and $c \sinh \xi$. The ratio of these axes is the aspect ratio $f=\tanh \xi$.

The case of the circle is obtained asymptotically for $c=0$ and $\xi=\infty$ with $\frac{c e^{\xi}}{2} \rightarrow R$, leading to $x_{1}=R \cos \eta$ and $x_{2}=R \sin \eta$. The metric tensor in the curvilinear frame is given by $g_{11}=g_{22}=c^{2} J^{2}$ with

$$
J^{2}=\frac{1}{2}(\cosh 2 \xi-\cos 2 \eta)
$$

The jacobian $J_{0}$ of the transformation from $(\xi, \eta)$ to $\left(x_{1}, x_{2}\right)$ is $J_{0}=c^{2} J^{2}$. All 315 points of an elliptic section can be obtained for $\eta$ between 0 and $2 \pi$ and $\xi$ between 0 and $\xi_{e}$, this last value being related to points on the boundary of the ellipse. 


\subsubsection{Solution of Helmholtz equation using elliptic coordinates}

The Helmholtz operator in elliptic coordinates is given (without body forces) by (Morse \& Feshbach, 1953):

$$
\Delta w_{3}+\frac{\omega^{2}}{v_{s}^{2}} w_{3}=\Delta w_{3}+k^{2} w_{3}=\frac{1}{c^{2} J^{2}}\left(\frac{\partial^{2} w_{3}}{\partial \xi^{2}}+\frac{\partial^{2} w_{3}}{\partial \eta^{2}}\right)+\frac{\omega^{2}}{v_{s}^{2}} u_{3}=0,
$$

where $v_{s}$ is the velocity of shear waves, with $v_{s}^{2}=\mu / \rho$, and $k_{s}=\omega / v_{s}$ is the 320 wave number.

The solution of this equation is found by separation of variables. The part related to the angular coordinate is solution of the historical Mathieu equation, used primarily to study the vibration of membranes (Mathieu, 1868):

$$
Y^{\prime \prime}(\eta)+\left[\Lambda-2 q_{s} \cos (2 \eta)\right] Y(\eta)=0,
$$

where $\Lambda$ is a separation constant and $q_{s}=\frac{1}{4} c^{2} k_{s}^{2}$. Similarly, the part related to the radial coordinate is solution of the modified Mathieu equation (Abramowitz \& Stegun, 1972):

$$
Z^{\prime \prime}(\xi)-\left[\Lambda-2 q_{s} \cosh (2 \xi)\right] Z(\xi)=0 .
$$

The solutions of the equation in $Y(\eta)$ are periodic only for discrete values of $\Lambda\left(q_{s}\right)$, known as "characteristic values". Knowing these characteristic values, the general periodic solution of Mathieu equation can be expanded along trigonometric functions as:

$$
w_{3}=\sum_{j=0}^{\infty} D_{j} c e_{j}\left(\eta, q_{s}\right) M c_{j}\left(\xi, q_{s}\right)+E_{j} s e_{j}\left(\eta, q_{s}\right) M s_{j}\left(\xi, q_{s}\right),
$$

where $c e_{j}$ (resp.se $e_{j}$ ) are obtained from a series of cosinus (resp. sinus) and $M c_{j}, M s_{j}$ are solutions of the modified Mathieu equation, named usually radial Mathieu functions. They are described below. Several notations and definitions can be used for these functions. Our notation refers to the definition of radial Mathieu functions introduced by Meixner \& Schäfke (1954), recalled thereafter.

As it will be seen thereafter, it is of prime importance to distinguish even and odd values of $j$, because they correspond to distinct symmetries of the solution. Finally, the different Mathieu functions found in the literature are given by: 


$$
\begin{aligned}
c e_{2 m}\left(\eta, q_{s}\right) & =\sum_{r=0}^{\infty} A_{2 r}^{(2 m)}\left(q_{s}\right) \cos 2 r \eta \\
s e_{2 m+2}\left(\eta, q_{s}\right) & =\sum_{r=0}^{\infty} B_{2 r}^{(2 m+2)}\left(q_{s}\right) \sin 2 r \eta \\
c e_{2 m+1}\left(\eta, q_{s}\right) & =\sum_{r=0}^{\infty} A_{2 r+1}^{(2 m+1)}\left(q_{s}\right) \cos (2 r+1) \eta \\
s e_{2 m+1}\left(\eta, q_{s}\right) & =\sum_{r=0}^{\infty} B_{2 r+1}^{(2 m+1)}\left(q_{s}\right) \sin (2 r+1) \eta .
\end{aligned}
$$

335 These functions have a period $\pi$ for even indices and a period $2 \pi$ for odd indices. The Fourier coefficients, for example $A_{2 r}^{(2 m)}\left(q_{s}\right)$, are related to specific characteristic values. Indeed, introducing the Fourier expansion of $c e_{2 m}\left(\eta, q_{s}\right)$ into equation (24), one obtains an infinite homogeneous system of linear equations. It can be expressed as an eigenvalue problem characterized by an infinite matrix(see Appendix A) : the eigenvalues of this matrix constitute the set of separation constants, denoted by $\Lambda=a_{2 m}$ and the components of the related eigenvectors are the Fourier coefficients $A_{2 r}^{(2 m)}$. Similarly, the separation constants related to $B_{2 r}^{(2 m+2)}$ (resp. $A_{2 r+1}^{(2 m+1)}, B_{2 r+1}^{(2 m+1)}$ ) are denoted by $\Lambda=b_{2 m}$ (resp. $\Lambda=a_{2 m+1}, b_{2 m+1}$ ). In the following, these Fourier coefficients will be considered as the terms of a square matrix $[A]$ (or $[B]$ ) and indexed by $r$ and $m$. Obtaining the characteristic values and the related Fourier coefficients has been the subject of numerous works. The numerical method used to obtain this set of coefficients is described in Appendix A, where the related references are recalled.

Different kinds of radial functions can be associated to these angular functions. However, it is recognized today that the best expressions for the radial functions are the ones using the series of products of Bessel functions introduced by Meixner \& Schäfke (1954). As an example, the radial function associated with $c e_{2 m}$ and corresponding to the kind of boundary conditions described 
previously is given by:

$M c_{2 m}(\xi)=\frac{1}{\varepsilon_{s} A_{2 s}^{(2 m)}} \sum_{k=0}^{\infty}(-1)^{k+m} A_{2 k}^{(2 m)}\left[J_{k-s}\left(u_{1}\right) J_{k+s}\left(u_{2}\right)+J_{k+s}\left(u_{1}\right) J_{k-s}\left(u_{2}\right)\right]$,

where $u_{1}=\sqrt{q_{s}} e^{-\xi}, u_{2}=\sqrt{q_{s}} e^{\xi}, \varepsilon_{s}=2$ when $s=0$ and $\varepsilon_{s}=1$ otherwise. $s$ is a "free" parameter. This parameter is often chosen arbitrarily as $s=0$ or $s=1$, depending on the type of radial function, but this choice is far from being optimal. A first reason is that $A_{2 s}^{(2 m)}\left(q_{s}\right)$ is outside the diagonal as soon as $m$ and $s$ are different. However, it is easy to see that for small values of $q_{s}$

35 (i.e. ellipses being almost a circle) the matrix $[A]$ is almost diagonal. So, this procedure leads to a division by $A_{2 s}^{(2 m)}\left(q_{s}\right)$ that is very small . From another point of view, it is of prime importance to check the procedure near the limit case of circular cylinders, where the solution is a simple expression of Bessel functions. A second reason is that this choice leads to very bad solutions when $m$ is increasing, as observed by Bibby \& Peterson (2013). These authors have shown that the best way to reduce the computational error for large values of $m$ is to choose the value of $s$ leading to the highest value of $\left|A_{2 s}^{(2 m)}\left(q_{s}\right)\right|$, for a given value of $m$. This choice, named "tuning method" by these authors, has been adopted in the following. More details are given in Appendix A.

\subsection{Eigenfrequencies for a longitudinal motion}

The eigenfrequencies are obtained by writing that the displacement is null on the boundary of the inclusion, leading to:

$$
w_{3}\left(\eta, \xi_{e}\right)=\sum_{p=0}^{\infty} D_{p} c e_{2 p}\left(\eta, q_{s}\right) M c_{2 p}\left(\xi_{e}, q_{s}\right)=0 .
$$

At this stage, it may be recalled that the Mathieu functions are orthogonal, complying with the following relations:

$$
\int_{0}^{2 \pi} c e_{2 p} c e_{2 q} d \eta=\pi \delta_{p q}
$$

where $\delta_{p q}$ is the Kronecker symbol. Multiplying the expansion of $w_{3}$ by $c e_{2 m}$ and integrating over $\eta$, one finds that $\pi M c_{2 m}\left(\xi_{e}, q\right)=0$. This shows that the 
370

each value of $m$, this equation has a set of solutions which are denoted by $q_{s(m)}^{(l)}$, where $l$ represents the indices of eigenfrequencies related to a given value of $m$.

These equations are solved, for a fixed aspect ratio, on a given frequency range, by splitting this frequency range into small intervals and looking for the intervals leading to a sign change of $M c_{2 m}\left(\xi_{e}, q_{s}\right)$. Next, the zero of this function over each found interval is obtained by using classical numerical methods.

\subsection{Participation factors}

The participation factors are given from a given eigenfunction $\mathbf{u}^{p}$ by:

$$
K_{j j}^{p}=\frac{\left(\left\langle\mathbf{u}^{p}\right\rangle_{(i)} \cdot \mathbf{e}_{j}\right)^{2}}{\left\langle\left\|\mathbf{u}^{p}\right\|^{2}\right\rangle_{(i)}}=\frac{I_{j}^{2}}{S \cdot M_{j}},
$$

where $S$ is the section of the fiber, given by $S=\pi . a . b, \mathbf{e}_{j}$ is a unit vector 380 along the direction of the macroscopic acceleration and $\langle\cdot\rangle_{(i)}$ is the average over the plane of the inclusion. The integrals over the section are:

$$
\begin{aligned}
I_{j} & =\int_{\Omega_{i}} \mathbf{u}^{p} \cdot \mathbf{e}_{j} d S \\
M_{j} & =\int_{\Omega_{i}}\left\|\mathbf{u}^{p}\right\|^{2} d S .
\end{aligned}
$$

For the case under consideration, $\mathbf{u}$ is parallel with $\mathbf{e}_{3}$ and these integrals are obtained by:

$$
\begin{aligned}
I_{3(m)}^{(l)} & =\int_{S} c e_{2 m}\left(\eta, q_{s(m)}^{(l)}\right) M c_{2 m}\left(\xi, q_{s(m)}^{(l)}\right) J_{0} d \xi d \eta \\
M_{3(m)}^{(l)} & =\int_{S}\left[c e_{2 m}\left(\eta, q_{s(m)}^{(l)}\right) M c_{2 m}\left(\xi, q_{s(m)}^{(l)}\right)\right]^{2} J_{0} d \xi d \eta
\end{aligned}
$$

\subsubsection{Computation of $I_{3}$}




$$
\begin{aligned}
I_{3(m)}^{(l)} & =\frac{c^{2}}{2}\left(T_{\eta 1(m)} U_{\xi 2(m)}-T_{\eta 2(m)} U_{\xi 1(m)}\right) \\
& =\frac{c^{2}}{2} I^{*}
\end{aligned}
$$

where

$$
\begin{aligned}
T_{\eta 1(m)} & =\int_{0}^{2 \pi} c e_{2 m} d \eta \\
T_{\eta 2(m)} & =\int_{0}^{2 \pi} c e_{2 m} \cos 2 \eta d \eta \\
U_{\xi 1(m)} & =\int_{0}^{\xi_{e}} M c_{2 m} d \xi \\
U_{\xi 2(m)} & =\int_{0}^{\xi_{e}} M c_{2 m} \cosh 2 \xi d \xi,
\end{aligned}
$$

all functions being computed for $q_{s}=q_{s(m)}^{(l)}$. The angular Mathieu functions are expanded along a trigonometric series:

$$
c e_{2 m}(\eta, q)=\sum_{r=0}^{\infty} A_{2 r}^{(2 m)}\left(q_{s}\right) \cos 2 r \eta .
$$

As a consequence, all terms of the expansion of the first two functions $T_{\eta 1(m)}$ and $T_{\eta 2(m)}$ can be obtained in a closed form. The related expressions are given in appendix C. For $U_{\xi 1(m)}$ and $U_{\xi 2(m)}$, they are computed by numerical integration over the segment $\left[0, \xi_{e}\right]$.

\subsubsection{Computation of $M_{3}$}

Similarly, $M_{3}$ can be obtained by

$$
\begin{aligned}
M_{3(m)}^{(l)} & =\frac{c^{2}}{2}\left(V_{\eta 1} W_{\xi 2}-V_{\eta 2} W_{\xi 1}\right) \\
& =\frac{c^{2}}{2} M^{*}
\end{aligned}
$$

with 


$$
\begin{aligned}
V_{\eta 1} & =\int_{0}^{2 \pi}\left(c e_{2 m}\right)^{2} d \eta \\
V_{\eta 2} & =\int_{0}^{2 \pi}\left(c e_{2 m}\right)^{2} \cos 2 \eta d \eta \\
W_{\xi 1} & =\int_{0}^{\xi_{e}}\left(M c_{2 m}\right)^{2} d \xi \\
W_{\xi 2} & =\int_{0}^{\xi_{e}}\left(M c_{2 m}\right)^{2} \cosh 2 \xi d \xi .
\end{aligned}
$$

As previously, the angular Mathieu function can be expanded into a trigonometric series, leading to the values given also in appendix $\mathrm{C}$. The radial integrals are obtained numerically.

\section{Solution for a plane motion}

\subsection{Solution for a macroscopic acceleration along $O x_{1}$}

The displacement field is solution of

$$
\begin{aligned}
\mu\left(\frac{\partial^{2} u_{1}}{\partial x_{1}^{2}}+\frac{\partial^{2} u_{1}}{\partial x_{2}^{2}}\right)+(\lambda+\mu)\left[\frac{\partial^{2} u_{1}}{\partial x_{1}^{2}}+\frac{\partial^{2} u_{2}}{\partial x_{1} \partial x_{2}}\right]+\rho \omega^{2}\left(u_{1}+U\right) & =0 \\
\mu\left(\frac{\partial^{2} u_{2}}{\partial x_{1}^{2}}+\frac{\partial^{2} u_{2}}{\partial x_{2}^{2}}\right)+(\lambda+\mu)\left[\frac{\partial^{2} u_{2}}{\partial x_{2}^{2}}+\frac{\partial^{2} u_{1}}{\partial x_{1} \partial x_{2}}\right]+\rho \omega^{2}\left(u_{2}\right) & =0
\end{aligned}
$$

where $-\omega^{2} U \mathbf{e}_{1}$ is the macroscopic acceleration. The structure of the problem leads to the following symmetries: $u_{1}$ is even in $x_{1}$ and $x_{2}$ and $u_{2}$ is odd in $x_{1}$ and $x_{2}$. The displacement can be obtained from the potentials $\varphi$ and $\Psi$ by:

$$
\mathbf{u}=\operatorname{grad} \varphi+\operatorname{curl} \Psi
$$

where $\boldsymbol{\Psi}=\psi \cdot \mathbf{e}_{3}$.

The previous symmetries are obtained as soon as $\varphi$ is odd in $x_{1}$ and even in $x_{2}$ and the reverse for $\psi$. Using elliptic coordinates, this can be achieved if

$$
\begin{aligned}
& \varphi \sim \sum \cos (2 m+1) \eta \\
& \psi \sim \sum \sin (2 m+1) \eta
\end{aligned}
$$


Thus, the potentials are given by

$$
\begin{aligned}
\varphi & =\sum E_{m} c e_{2 m+1}\left(\eta, q_{p}\right) M c_{2 m+1}\left(\xi, q_{p}\right) \\
\psi & =\sum F_{m} s e_{2 m+1}\left(\eta, q_{s}\right) M s_{2 m+1}\left(\xi, q_{s}\right),
\end{aligned}
$$

where $E_{m}$ and $F_{m}$ are constant. $q_{p}$ and $q_{s}$ are given by:

$$
\begin{aligned}
q_{p} & =\frac{1}{4} c^{2} \frac{\omega^{2}}{v_{p}^{2}}=k_{c}^{2} q_{s} \\
q_{s} & =\frac{1}{4} c^{2} \frac{\omega^{2}}{v_{s}^{2}},
\end{aligned}
$$

405 where the ratio of celerities is denoted by $k_{c}=\frac{v_{s}}{v_{p}}=\sqrt{\frac{1-2 \nu}{2(1-\nu)}}$.

From these potentials, the displacement field is obtained by (Eringen \& Suhubi, 1978):

$$
\begin{aligned}
& u_{\xi}=\frac{1}{c J}\left(\frac{\partial \varphi}{\partial \xi}+\frac{\partial \psi}{\partial \eta}\right) \\
& u_{\eta}=\frac{1}{c J}\left(\frac{\partial \varphi}{\partial \eta}-\frac{\partial \psi}{\partial \xi}\right)
\end{aligned}
$$

Finally, the displacement field is given by:

$$
\begin{aligned}
(c J) u_{\xi}= & \sum_{m} E_{m} c e_{2 m+1}\left(\eta, q_{p}\right) C e_{2 m+1}^{\prime}\left(\xi, q_{p}\right) \\
& +\sum_{m} F_{m} s e_{2 m+1}^{\prime}\left(\eta, q_{s}\right) S e_{2 m+1}\left(\xi, q_{s}\right) \\
(c J) u_{\eta}= & \sum_{m} E_{m} c e_{2 m+1}^{\prime}\left(\eta, q_{p}\right) C e_{2 m+1}\left(\xi, q_{p}\right) \\
& -\sum_{m} F_{m} s e_{2 m+1}\left(\eta, q_{s}\right) S e_{2 m+1}^{\prime}\left(\xi, q_{s}\right),
\end{aligned}
$$

where $F^{\prime}$ is the derivative of $F$ with respect to $\xi$ (or $\eta$ ).

As seen in the previous section, the angular Mathieu functions are known through their expansion in Fourier series. Introducing this Fourier expansion into the displacement field leads to:

$$
(a J) u_{\xi}=\sum_{r=0}^{\infty} \cos (2 r+1) \eta H_{r}(\xi, \omega)
$$


410

where $H_{r}$ is given by:

$$
\begin{aligned}
& H_{r}(\xi, \omega)= \sum_{m} E_{m}\left[A_{2 r+1}^{(2 m+1)}\left(q_{p}\right)\right] C e_{2 m+1}^{\prime}\left(\xi, q_{p}\right) \\
&+\sum_{m} F_{m}\left[B_{2 r+1}^{(2 m+1)}\left(q_{s}\right)(2 r+1)\right] S e_{2 m+1}\left(\xi, q_{s}\right) .
\end{aligned}
$$

Similarly,

$$
(a J) u_{\eta}=\sum_{t=0}^{\infty} \sin (2 t+1) \eta K_{t}(\xi, \omega)
$$

with

$$
\begin{gathered}
K_{t}(\xi, \omega)=-\sum_{m} E_{m}\left[A_{2 t+1}^{(2 m+1)}\left(q_{p}\right)(2 t+1)\right] C e_{2 m+1}\left(\xi, q_{p}\right) \\
-\sum_{m} F_{m}\left[B_{2 t+1}^{(2 m+1)}\left(q_{s}\right)\right] S e_{2 m+1}^{\prime}(\xi, q s) .
\end{gathered}
$$

Finally, functions $H$ and $K$ are obtained by the matrix product

$$
\left[\begin{array}{l}
H \\
K
\end{array}\right]=\left[\begin{array}{ll}
M^{11} & M^{12} \\
M^{21} & M^{22}
\end{array}\right]\left[\begin{array}{c}
E \\
F
\end{array}\right]=[M(\omega, \xi)]\left[\begin{array}{c}
E \\
F
\end{array}\right],
$$

where the submatrices can be obtained from the Fourier coefficients of Mathieu functions and from the radial Mathieu functions by the following relations:

$$
\begin{aligned}
M_{r m}^{11} & =A_{2 r+1}^{(2 m+1)}\left(q_{p}\right) C e_{2 m+1}^{\prime}\left(\xi, q_{p}\right) \\
M_{r m}^{12} & =B_{2 r+1}^{(2 m+1)}\left(q_{s}\right)(2 r+1) S e_{2 m+1}\left(\xi, q_{s}\right) \\
M_{t m}^{21} & =-A_{2 t+1}^{(2 m+1)}\left(q_{p}\right)(2 t+1) C e_{2 m+1}\left(\xi, q_{p}\right) \\
M_{t m}^{22} & =-B_{2 t+1}^{(2 m+1)}\left(q_{s}\right) S e_{2 m+1}^{\prime}\left(\xi, q_{s}\right) .
\end{aligned}
$$

Compared with the antiplane case, it can be seen that the solution couples now all Mathieu functions and their derivatives.

\subsubsection{Computation of the eigenfrequencies}

The eigenfrequencies are obtained for a non null displacement vanishing at the boundary. It leads to the search of frequencies $\omega_{l}$ being such that $[M]$ is singular, or $\operatorname{det}\left(\left[M\left(\omega_{l}, \xi_{e}\right)\right]\right)=0$. Compared with the case of the previous section, it can be seen that it is no more possible to distinguish the eigenfrequencies related to separate values of $m$. Now, each eigenfrequency involves all values of $m$ simultaneously. 


\subsubsection{Computation of the participation factors}

The participation factors are obtained from the integrals over the elliptic section of the components of the displacement field by:

$$
\begin{aligned}
I_{2}^{(l)} & =\int_{\Omega_{i}} \mathbf{u}^{(l)} \cdot \mathbf{e}_{1} d S \\
& =\int_{\Omega_{i}}\left(u_{\xi}^{(l)} \mathbf{e}_{\xi}+u_{\eta}^{(l)} \mathbf{e}_{\eta}\right) \cdot \mathbf{e}_{1} d S \\
& =\int_{\Omega_{i}}\left(u_{\xi}^{(l)} \mathbf{e}_{\xi} \cdot \mathbf{e}_{1}+u_{\eta}^{(l)} \mathbf{e}_{\eta} \cdot \mathbf{e}_{1}\right) d S \\
M_{2}^{(l)} & =\int_{\Omega_{i}}\left\|\mathbf{u}^{(l)}\right\|^{2} d S .
\end{aligned}
$$

It involves the projections of the local unit vectors on $\mathbf{e}_{1}$ given by:

$$
\begin{aligned}
\mathbf{e}_{\xi} \cdot \mathbf{e}_{1} & =\frac{\sinh \xi \cos \eta}{J} \\
\mathbf{e}_{\eta} \cdot \mathbf{e}_{1} & =-\frac{\cosh \xi \sin \eta}{J} .
\end{aligned}
$$

Taking into account the expansion of the components of the displacement fields leads to the expression of the participation factors:

$$
K_{22}^{(l)}=\frac{\left[\int_{0}^{\xi_{e}}\left[H_{0}\left(\xi, \omega_{l}\right) \sinh \xi d \xi-K_{0}\left(\xi, \omega_{l}\right) \cosh \xi\right] d \xi\right]^{2}}{\sinh \xi_{e} \cosh \xi_{e} \int_{0}^{\xi_{e}}\left(\sum_{r} H_{r}^{2}\left(\xi, \omega_{l}\right)+\sum_{t} K_{t}^{2}\left(\xi, \omega_{l}\right)\right) d \xi} .
$$

The steps are similar to the previous case. Obviously, the symmetries are changed, $\varphi$ being now even in $x_{1}$ and odd in $x_{2}$ and the reverse for $\psi$, leading to:

$$
\begin{aligned}
\psi & =\sum F_{m} c e_{2 m+1}\left(\eta, q_{s}\right) C e_{2 m+1}\left(\xi, q_{s}\right) \\
\varphi & =\sum E_{m} s e_{2 m+1}\left(\eta, q_{p}\right) S e_{2 m+1}\left(\xi, q_{p}\right) .
\end{aligned}
$$

It leads to the components of the displacement field:

$$
\begin{aligned}
& (a J) u_{\xi}=\quad \sum_{r=0}^{\infty} \sin (2 r+1) \eta N_{r}(\xi, \omega) \\
& (a J) u_{\eta}=\sum_{r=0}^{\infty} \cos (2 r+1) \eta Q_{r}(\xi, \omega),
\end{aligned}
$$


where $N_{r}$ and $Q_{r}$ are:

$$
\begin{array}{cc}
N_{r}(\xi, \omega)= & \sum_{m} E_{m} B_{2 r+1}^{(2 m+1)}\left(q_{p}\right) S e_{2 m+1}^{\prime}\left(\xi, q_{p}\right) \\
& -\sum_{m} F_{m} A_{2 r+1}^{(2 m+1)}\left(q_{s}\right) \cdot(2 r+1) C e_{2 m+1}\left(\xi, q_{s}\right) \\
Q_{r}(\xi, \omega)= & \sum_{m} E_{m} B_{2 r+1}^{(2 m+1)}\left(q_{p}\right)(2 r+1) S e_{2 m+1}\left(\xi, q_{p}\right) \\
& -\sum_{m} F_{m} A_{2 r+1}^{(2 m+1)}\left(q_{s}\right) C e_{2 m+1}^{\prime}\left(\xi, q_{s}\right) .
\end{array}
$$

Finally, the $N_{r}$ (resp. $Q_{r}$ ) are the components of $[N]$ (resp. $[Q]$ ) given by

$$
\left[\begin{array}{l}
N \\
Q
\end{array}\right]=\left[\begin{array}{ll}
T^{11} & T^{12} \\
T^{21} & T^{22}
\end{array}\right]\left[\begin{array}{c}
E \\
F
\end{array}\right]
$$

where the components of matrix $[T]$ are obtained from

$$
\begin{aligned}
T_{r m}^{11} & =B_{2 r+1}^{(2 m+1)}\left(q_{p}\right) S e_{2 m+1}^{\prime}\left(\xi, q_{p}\right) \\
T_{r m}^{12} & =-A_{2 r+1}^{(2 m+1)}\left(q_{s}\right)(2 r+1) C e_{2 m+1}\left(\xi, q_{s}\right) \\
T_{r m}^{21} & =B_{2 r+1}^{(2 m+1)}\left(q_{p}\right)(2 r+1) S e_{2 m+1}\left(\xi, q_{p}\right) \\
T_{r m}^{22} & =-A_{2 r+1}^{(2 m+1)}\left(q_{s}\right) C e_{2 m+1}^{\prime}\left(\xi, q_{s}\right) .
\end{aligned}
$$

As before, the eigenfrequencies $\omega_{l}$ cancel the determinant of $\left[T\left(\omega_{l}, \xi_{e}\right)\right]$.

The participation factors are obtained similarly and given by:

$$
K_{33}^{(l)}=\frac{\left[\int_{0}^{\xi_{e}}\left[N_{0}\left(\xi, \omega_{l}\right) \cosh \xi+Q_{0}\left(\xi, \omega_{l}\right) \sinh \xi\right] d \xi\right]^{2}}{\sinh \xi_{e} \cosh \xi_{e} \sum_{r=0}^{\infty} \int_{0}^{\xi_{e}}\left[N_{r}^{2}\left(\xi, \omega_{l}\right)+Q_{r}^{2}\left(\xi, \omega_{l}\right)\right] d \xi} .
$$

\section{The extremal cases}

The main geometrical parameter of the problem related to elliptical inclusions is the aspect ratio corresponding to the ratio between the radii of the elliptic section. From a physical point of view, changing the aspect ratio from 1 to small values corresponds to the path from circular fibers to very flat fibers, 445 i.e. a stratified medium made of a stiff matrix containing very large parallel soft plates of finite thickness. So, it is important for the interpretation of the numerical results to know the eigenvalues and participation factors in these limit cases. The results related to these limit cases are recalled below. 


\subsection{Fibers of circular cross section}

450

$R$ were obtained in Bonnet \& Monchiet (2017). The non-dimensional eigenfrequencies obtained in this paper defined by $\omega^{*}=\omega R / v_{s}$, are the zeros of Bessel function $J_{0}$ for a longitudinal motion and the solutions of an equation involving Bessel functions of orders 0 and 1 for a transversal motion.

The eigenfrequencies related to Bessel functions of higher order were discarded in Bonnet \& Monchiet (2017) because they lead to null participation factors in the case of the circle. However, it will be seen thereafter that the limit cases of eigenfrequencies for almost circular elliptic fibers contain also eigenfrequencies that can be related to Bessel functions of higher orders.

Finally these limit cases are the solutions of $J_{2 m}=0$ for a longitudinal macroscopic acceleration and the solutions of

$$
\begin{gathered}
-k_{c} \omega^{*} J_{2 m}\left(k_{c} \omega^{*}\right) J_{2 m}\left(\omega^{*}\right)+k_{c}(2 m+1) J_{2 m}\left(k_{c} \omega^{*}\right) J_{2 m+1}\left(\omega^{*}\right) \\
+(2 m+1) J_{2} m\left(\omega^{*}\right) J_{2 m+1}\left(k_{c} \omega^{*}\right)=0,
\end{gathered}
$$

for a transversal macroscopic acceleration. Participation factors are null for $m \neq 0$ and given by explicit expressions in Bonnet \& Monchiet (2017) for $m=0$.

\subsection{Very flat fibers}

465

In this case, the section of the fibers corresponds to an ellipse of minor radius $b$ and a very large major radius. It can be assimilated to the zone comprised between two parallel planes distant from $2 b$. The non-dimensional eigenfrequencies are again defined by $\omega^{*}=\omega b / v_{s}$. For a motion parallel to the mean plane they are equal to $\omega_{n}^{*}=\pi / 2+n . \pi$ and the participation factors are given by ${ }_{470} K_{n}=2 / \omega^{* 2}$.

For a motion perpendicular to the mean plane of the inclusions, the eigenfrequencies are given by $\omega_{n}^{*}=(\pi / 2+n \pi) / k_{c}$ and the participation factors by $K_{n}=2 k_{c}^{2} / \omega^{* 2}$. 


\section{Numerical results}

\subsection{Longitudinal motion}

For the longitudinal motion, the solution does not involve compressional waves. As a consequence, the eigenfrequencies are functions of the shear modulus $\mu$ and of the radii $a$ and $b$ of the fibers. All results can be expressed by looking for the non-dimensional frequency $\omega^{*}=b \omega / v_{s}$ as a function of the aspect ratio $f=b / a$. The length introduced into the non dimensional frequency is the smaller radius of the ellipse. The aspect ratios related to decreasing values of $f$ correspond to ellipses of the same smaller radius and increasing values of the larger radius.

As explained in section 3, the modes related to Mathieu functions of different orders are decoupled. So, for each value of order $m$, one can find a set of eigenfrequencies which cancel the value of the radial Mathieu functions at the surface of the fiber.

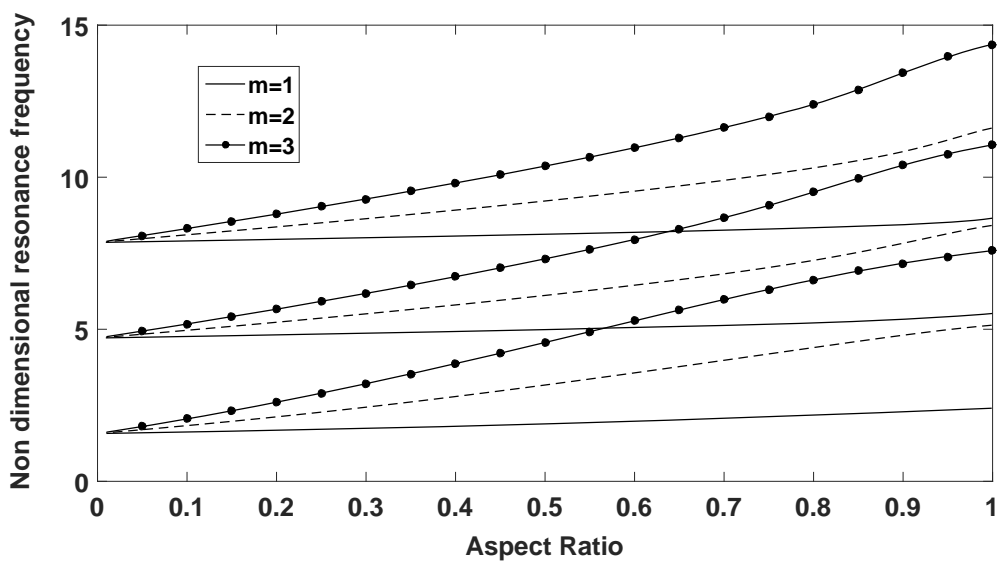

Figure 2: Resonance frequencies for a longitudinal motion as a function of the aspect ratio: modes related to the first three eigenfrequencies for Mathieu functions of first three orders.

Fig. 2 presents the first three eigenfrequencies of the first three values of $m$. It has been checked that, for the limit case of circular cross sections $(f=$ 490 1), the results recover the values of eigenfrequencies for $m=1$ obtained in Bonnet \& Monchiet (2017). The values for $m>1, f=1$ recover precisely the 
ones obtained for circular fibers and higher indices of Bessel functions given in the previous section. The limit case of small aspect ratio is also of interest. It can be seen that the first eigenfrequencies for $m=0,1,2$ tend to $\pi / 2$ while the second (resp. third) eigenfrequencies tend to $3 \pi / 2$ (resp. $5 \pi / 2$ ), these limit values corresponding to the case of very flat fibers recalled in the previous section.

All eigenfrequencies increase with $f$, due to the fact that the section of the ellipse decreases with $f$ when $b$ is constant.

The participation factors that have a significant contribution related to the eigenfrequencies of Fig. 2 are reported in Fig. 3. It can be seen for almost circular sections that the participation factors are null for $m>0$ and are not null for $m=0$, which corresponds to the results obtained in Bonnet \& Monchiet (2017). However, the curves show that the participation factors increase for smaller aspect ratios, the contribution for the first eigenfrequencies related to $m>0$ becoming significative. For small aspect ratios, it is no more possible to compare the participation factors with those of the plane inclusions. Indeed, the first participation factor for the plane inclusions must now be compared with the sum of all first participation factors for all values of $m$.

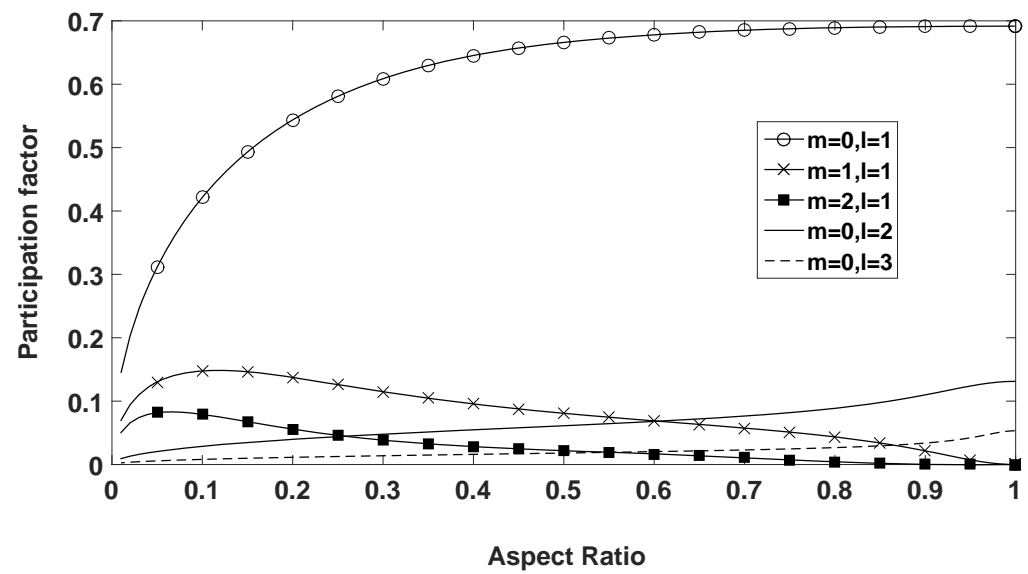

Figure 3: Participation factors for a longitudinal motion as a function of the aspect ratio: modes related to the 9 cases of Fig. 2 which have a significant contribution. 


\subsection{Transversal motion}

In the case of a transversal motion, compressional waves and shear waves are simultaneously involved in the solution. As a consequence, the results depend not only on the non dimensional frequency, but also on the Poisson's ratio.

\subsubsection{Effect of the aspect ratio}

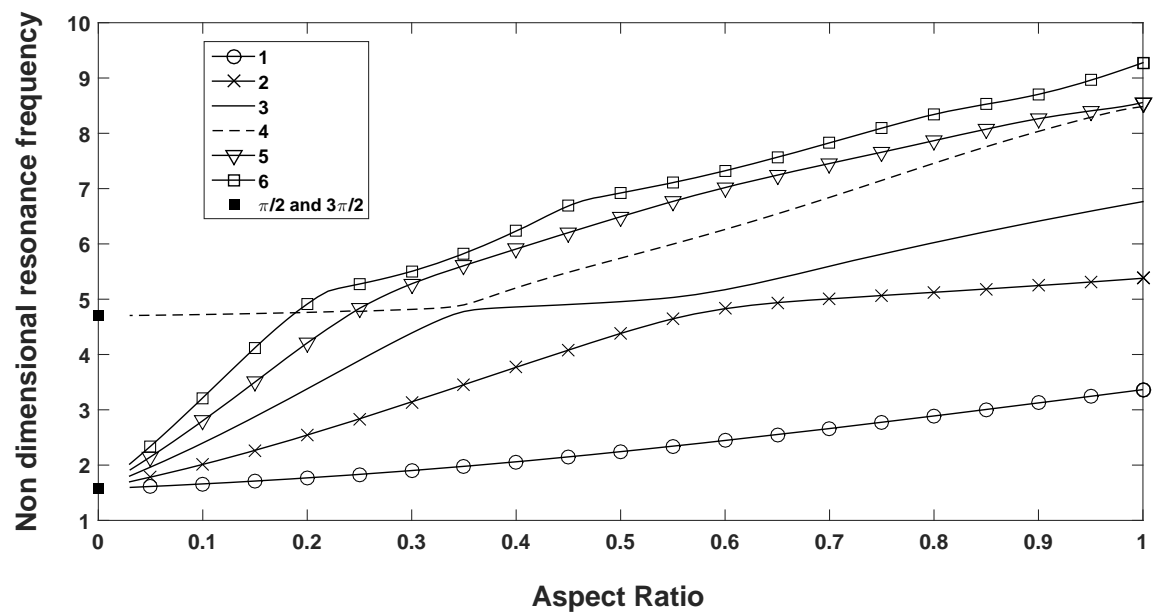

Figure 4: Resonance frequencies for a transversal motion along $x_{1}$ as a function of the aspect ratio for $\nu=0.25$ : six first modes .

The results have been obtained in the case of an average value of the Poisson's ratio equal to 0.25 . The first six eigenfrequencies related to the motion along $x_{1}$ are reported in Fig. 4. Contrarily to the case of the longitudinal motion, the Mathieu functions of any order contribute to the motion. So, it is no more possible to separate the different modes as previously. The values related to $f$ near 1 tend to the 6 first eigenfrequencies for the transversal motion of the circular cylinder. For small values of $f$, five of the non dimensional eigenfrequencies tend to $\pi / 2$, while the fourth eigenfrequency tends to $3 \pi / 2$, recovering again the first eigenfrequencies for the plane inclusions. It can be seen that the curve related to the fourth eigenfrequency crosses the one corresponding to eigenfrequencies 5 and 6 . This induces a complication in the numerical computation. Indeed, for the case of longitudinal motion, the computation of 


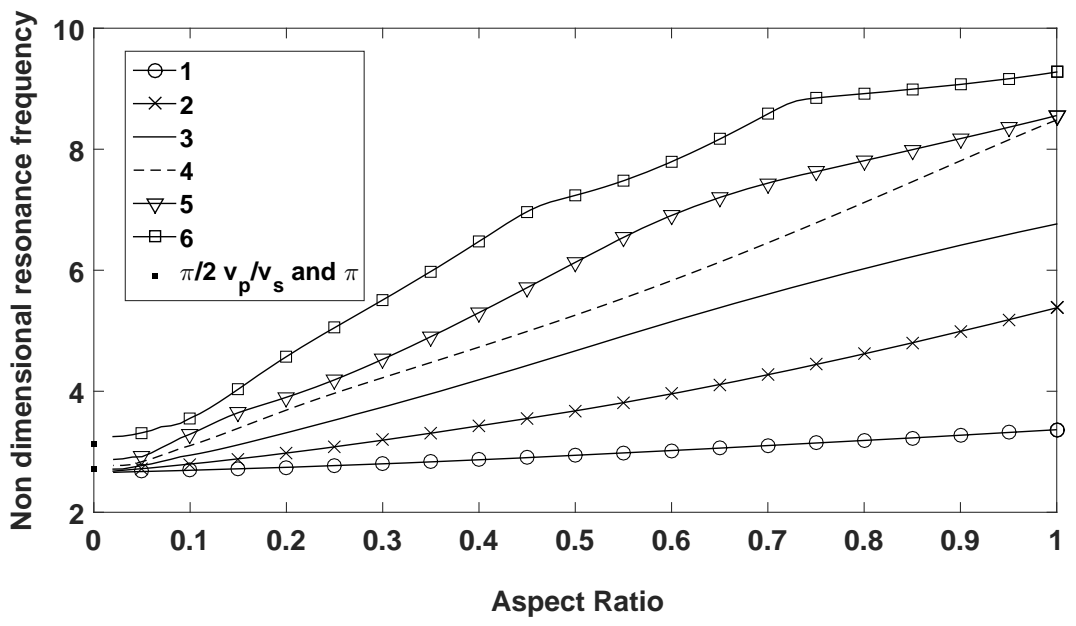

Figure 5: Resonance frequencies for a transversal motion along $x_{2}$ as a function of the aspect ratio for $\nu=0.25$ : six first modes .

all eigenfrequencies are decoupled, while in the case of transversal motion, all results are obtained from a fully coupled system. So, the curves were obtained by starting from the eigenfrequency for the circle. Next the eigenfrequencies for decreasing values of $f$ are obtained by looking for decreasing frequencies with the use of a prolongation algorithm for $f<0.95$.

The first six eigenfrequencies for the motion along $x_{2}$ are reported in Fig. 5 The values for $f=1$ are obviously identical to the ones obtained in the previous case. The feature of the results is significantly different from the previous case for smaller aspect ratios. The first five eigenfrequencies are close to $\frac{\pi}{2} \frac{v_{p}}{v_{s}}$ : this corresponds to the first eigenfrequency for the plane inclusions. However, the sixth eigenfrequency is close to the value $\omega^{*}=\pi$ instead of the second eigenfrequency for the stratified medium. It can be interpreted as follows: as seen in section 3 , in this case, the component $u_{1}$ is odd in $x_{1}$ and $x_{2}$. This can be achieved if the variation of $u_{1}$ along $x_{2}$ is such that $u_{1} \sim \sin \left(\omega x_{2} / c_{s}\right)$. The condition at the surface of the plane inclusion is achieved by $\omega^{*}=\omega b / c_{s}=\pi$, which can explain the limit value obtained for the sixth eigenfrequency when $f$ is close to 0 . 


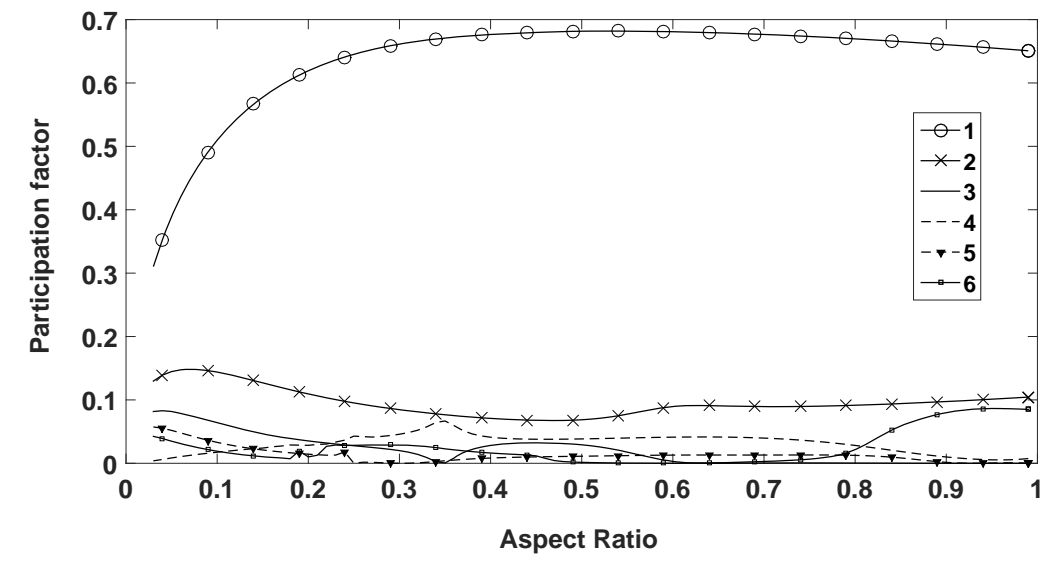

Figure 6: Participation factors for a transversal motion along $x_{1}$ as a function of the aspect ratio for $\nu=0.25$ : six first modes .

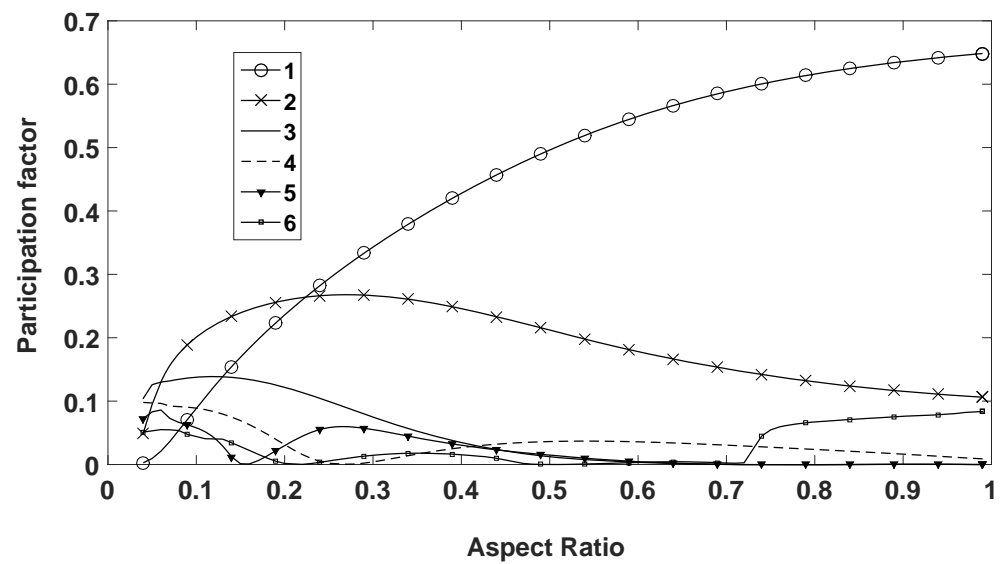

Figure 7: Participation factors for a transversal motion along $x_{2}$ as a function of the aspect ratio for $\nu=0.25$ : six first modes.

The participation factors for the motion along $x_{1}$ are given in Fig. 6. It has been checked that the values obtained for $f=1$ are the ones obtained for the circular cylinder. It can be seen that the first participation factor is largely dominant, except for small aspect ratios where this factor decreases significantly. In addition, the fourth and third curves have opposite trends for $f$ around 0.35 . This corresponds to the point where the third and fourth eigenfrequencies are 
almost the same on Fig. 4. In this case, the eigenfrequencies are no more distinct: there is only one eigenfrequency whose participation factor is the sum of the values obtained on the two curves. The crossings of curve 4 with curves 5 and 6 lead to similar perturbations of the general trend.

The participation factors for the motion along $x_{2}$ are reported in Fig. 7 The participation factor of the first mode is now decreasing drastically and is close to 0 for small aspect ratios, while the second participation factor reaches almost 0.3 around $f=0.25$ and becomes the major contribution to the motion.

\subsubsection{Effect of the Poisson's ratio}

The eigenfrequencies have been computed for a mean value of aspect ratio $f=0.5$ and increasing values of the Poisson's ratio up to 0.48 . The results

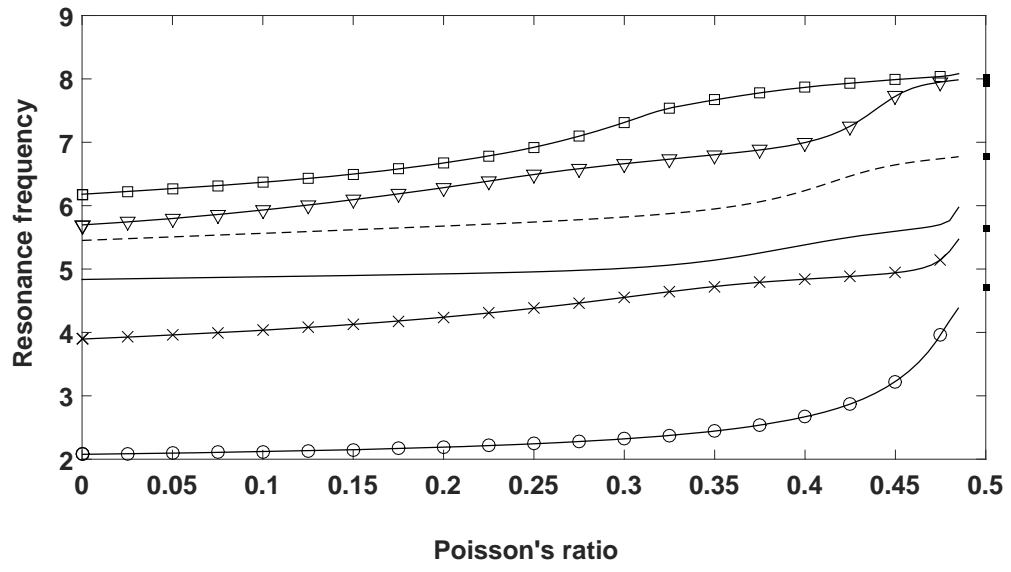

Figure 8: Resonance frequencies for a transversal motion along $x_{1}$ as a function of the Poisson's ratio for an aspect ratio $f=0.5$ : six first modes. The squares at $\nu=0.5$ are the values for the strictly incompressible case.

corresponding to the motion along $x_{1}$ are reported in Fig. 8, All resonance frequencies increase with $\nu$; the increase is moderate up to 0.4 and becomes stronger when $\nu$ is close to 0.5 (which corresponds to an incompressible material) more specifically for the first eigenfrequency. The points reported at $\nu=0.5$ have been obtained by solving the case of a strictly incompressible material, because the process described in section 4 can no more be used when $\nu$ is close 
to 0.5 , the ratio of the celerities becoming infinite. The solution is similar to the compressible case, except that the dynamic term in the dynamic equation for the potential $\varphi$ tends to 0 . As a consequence, $\varphi$ is the solution of Laplace equation and is therefore a static, harmonic potential. $\psi$ is still the solution of scalar Helmholtz equation. The results are compatible with those obtained in the compressible case taking into account that the third and fourth eigenfrequencies for the incompressible case are almost the same. In the case of the motion along

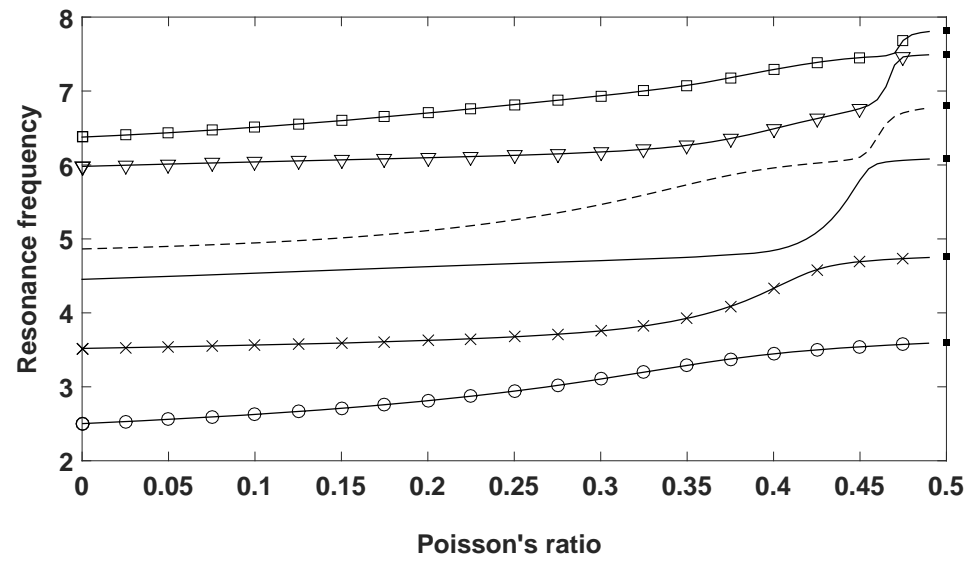

Figure 9: Resonance frequencies for a transversal motion along $x_{2}$ as a function of the Poisson's ratio for an aspect ratio $f=0.5$ : six first modes. The squares are obtained for a strictly incompressible material.

$x_{2}$, reported in Fig. 9, similar trends are observed, but the steepest part of the curve is near $\nu=0.47$, the curve being almost horizontal at $\nu=0.5$, the values being nearly the same as for the incompressible case in the last part of the curve.

The participation factors for the case of motion along $x_{1}$ given in Fig. 10 show that the Poisson's ratio affects sligthly the participation factors up to $\nu=0.3$. The curve for the first mode decreases to zero between 0.4 and 0.5 while the curves for higher modes show a maximum for Poisson's ratios increasing with the order of the mode and tend to zero for $\nu=0.5$, recovering a null participation factor for the case of incompressibility, similarly to the case of circular fibers 


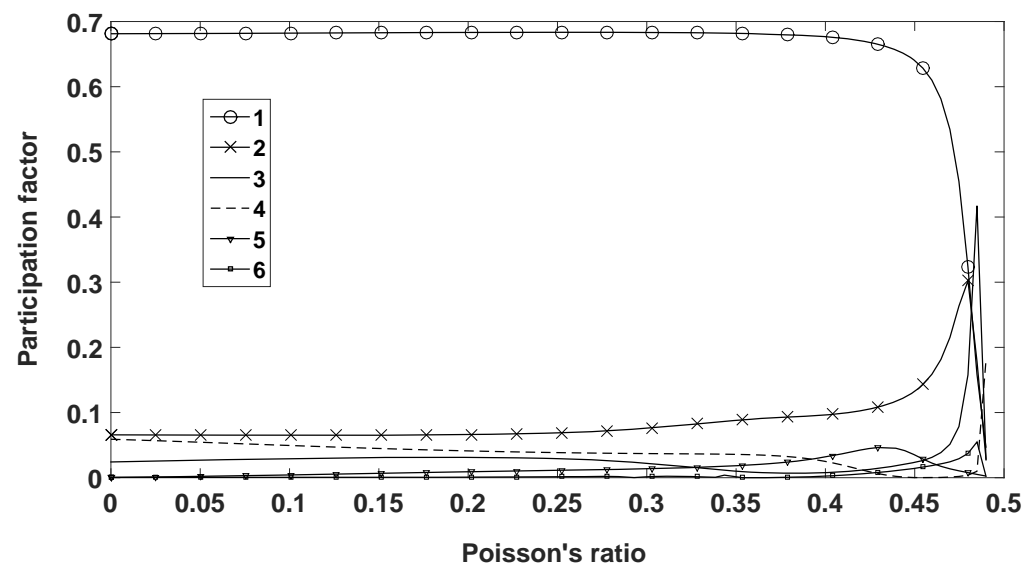

Figure 10: Participation factors for a transversal motion along $x_{1}$ as a function of the Poisson's ratio for an aspect ratio $f=0.5$ : six first modes .

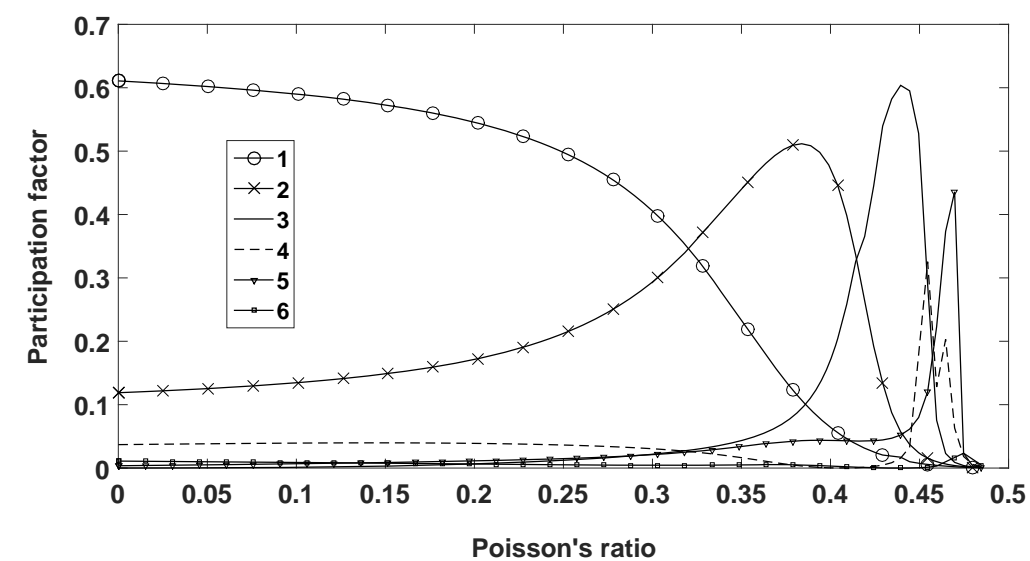

Figure 11: Participation factors for a transversal motion along $x_{2}$ as a function of the Poisson's ratio for an aspect ratio $f=0.5$ : six first modes .

(Bonnet \& Monchiet, 2017). For the case of the motion along $x_{2}$, the decrease of the participation factor of the first mode appears for lower values of $\nu$, the participation factor of the second mode being more important for $\nu>0.3$. All modes greater than 2 display a peak at values of Poisson's ratios smaller than in the case of the motion along $x_{1}$. 


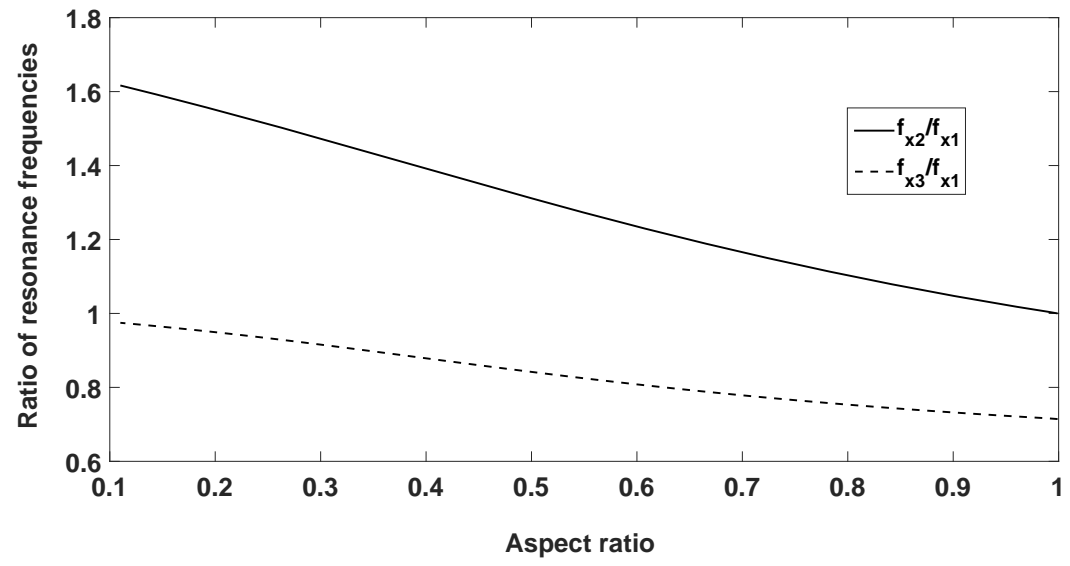

Figure 12: Ratios of first resonance frequencies for different directions as a function of the aspect ratio for $\nu=0.25$.

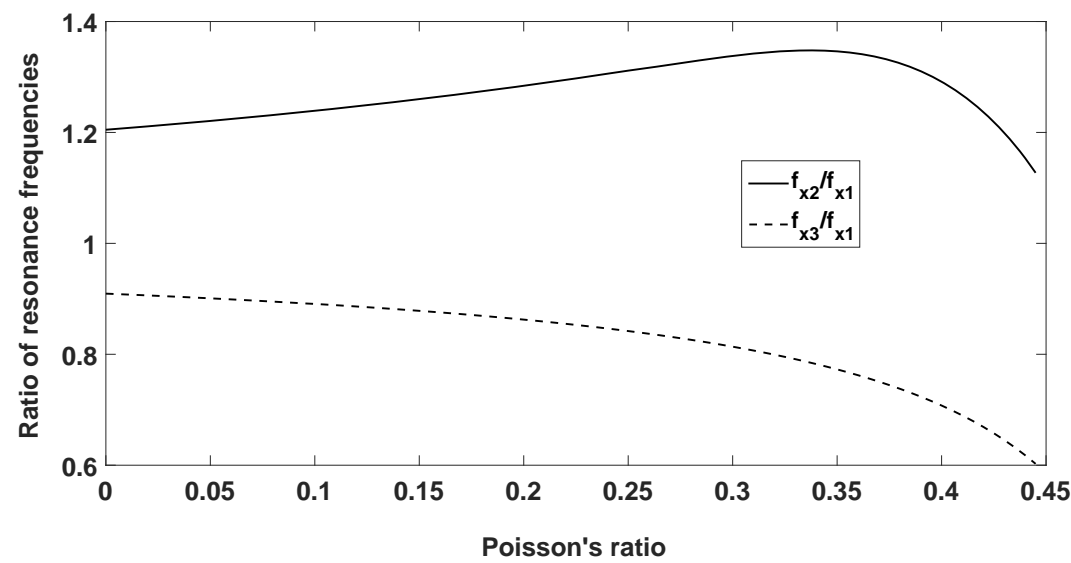

Figure 13: Ratio of resonance frequencies for different directions as a function of the Poisson's ratio for $f=0.5$.

\subsection{Anisotropy effect}

One important aspect of composites with elliptic fibers is that the dynamic density is fully orthotropic, its values being all different along the axes of symmetry. This anisotropy is characterized also by the fact that the material displays stop frequency bands that are different along these axes. From another point of view, it has been found that the participation factor of the first mode dominates 
for a large range of Poisson's ratios and aspect ratios. So, denoting by $f_{x_{i}}$ the of acoustic waves by suitable metamaterials will also be of interest. 


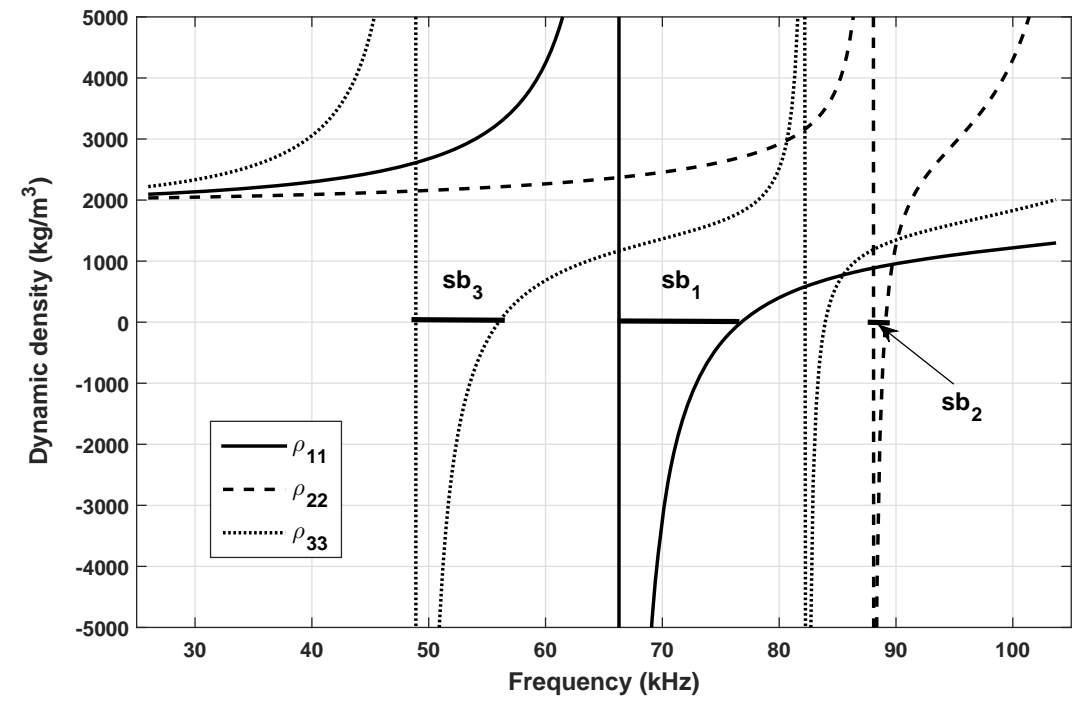

Figure 14: The three components of dynamic density for a composite of aluminium matrix and PVC fibers with an aspect ratio of 0.5. The first stop band for each direction $i$ is reported by $s b_{i}$

The most popular acoustic metamaterials, are made of composite spheres. In this case, the dynamic density is isotropic. They act as "spring-mass resonators" but not as polarizers, because the stop bands are characterized by the same frequency ranges for all directions. It means that no wave is transmitted at these stop bands. By comparison, fiber composites act fully as polarizers. Compared with optical polarizers a main difference comes from the kind of polarization that can be obtained: electromagnetic waves are all transversal ones and the linear polarization restricts only the direction of the transverse electric field to be parallel to a specific axis. For acoustic waves in solids, there are transversal (shear) waves and longitudinal (compressional) waves. So, the polarization can affect each one of these waves.

Taking into account the results obtained from the previous sections for elliptic fibers, the polarizing effect of the composite for different directions of propagation is given in table 1 . It seems more suitable to build polarizers made 


\begin{tabular}{|c|c|c|c|c|c|}
\hline & \multicolumn{3}{|c|}{ Elliptical } & \multicolumn{2}{c|}{ Circular } \\
\hline Resonance frequencies $\rightarrow$ & $f_{1}$ & $f_{2}$ & $f_{3}$ & $f_{1}=f_{2}$ & $f_{3}$ \\
\hline Propagagation along $\downarrow$ & & & & & \\
\hline$x_{1}$ & $\mathrm{~S}_{2} \mathrm{~S}_{3}$ & $\mathrm{PS}_{3}$ & $\mathrm{PS}_{2}$ & $\mathrm{~S}_{3}$ & $\mathrm{PS}_{2}$ \\
\hline$x_{2}$ & $\mathrm{PS}_{3}$ & $\mathrm{~S}_{1} \mathrm{~S}_{3}$ & $\mathrm{PS}_{1}$ & $\mathrm{~S}_{3}$ & $\mathrm{PS}_{1}$ \\
\hline
\end{tabular}

Table 1: The polarization effect of composites made of circular or elliptic fibers for a propagation transverse to the direction of the fibers. The table provides the polarization of the waves obtained after crossing the composite for waves whose spectral contents are within the stop bands at $f_{1}, f_{2}, f_{3}$. For example, $P S_{2}$ means that the transmitted plane wave is characterized by the components of displacement corresponding to a $P$ wave and a shear wave $S_{2}$ polarized along $x_{2}$.

of plates of metamaterials, the directions of propagation being perpendicular to the axes of the fibers. Therefore, the direction of propagation has been restricted to $x_{1}$ and $x_{2}$. For the sake of comparison, the case of circular fibers is also reported. For elliptic fibers, it can be seen that the choice of frequency range allows us to turn off separately any direction of polarization, while in the case of circular waves it is possible to turn off either one direction of polarization or simultaneously two directions of polarization in the plane of the section of the cylinder. The circular fibers allow us to obtain shear waves polarized along only one direction, similarly to the case of optical polarization.

Finally, the use of fibers with elliptical or circular cross sections are complementary in the design of acoustic polarizers.

\section{Conclusion}

Composites with a large contrast can be locally resonant under conditions on elastic properties and densities specified in section 2. The dynamic density is a function of the resonance frequencies of the fibers and of the participation factors related to the resonance frequencies. Composites containing fibers of elliptic cross sections have been studied: the dynamic density is fully orthotropic 
in this case, its components being different for different directions of motion along the axes of symmetry of the fiber. The computation of resonance frequencies and participation factors has been effected by solving the plane dynamic problems with the help of Mathieu functions. It needs a significant effort to use these functions, specially for small aspect ratios. The tuning method of Bibby \& Peterson (2013) has been very helpful in this work.

660

The computation of resonance frequencies is relatively easy in the case of a motion along the direction of fibers. They are given by the zeros of radial Mathieu functions. For transversal motions, the eigenfrequencies are obtained from a linear system coupling all radial Mathieu functions.

The non dimensional resonance frequencies are affected by the aspect ratio of the elliptical section and by the Poisson's ratio. They decrease continuously with decreasing aspect ratio from the values related to the circular cylinder to the ones related to very flat inclusions when the aspect ratio becomes very small. The effect of Poisson's ratio on resonance frequencies is moderate except at the vicinity of $\nu=0.5$ corresponding to the strictly incompressible case.

The participations factors corresponding to the first eigenfrequency are the main ones for a large set of aspect ratios and Poisson's ratios. However, they becomes inferior to the ones related to the second eigenfrequency for small aspect ratios in the case of the motion along $x_{2}$. The participation factors at increasing orders display a maximum near $\nu=0.5$, but are all null for strictly incompressible materials. It means that the participation factors present strong perturbations near $\nu=0.5$ and that the conception of such metamaterials does not seem feasible for almost incompressible fibers.

The anisotropy of the dynamic density, characterized by the eigenfrequencies related to different directions of motion, is moderate, the ratio of eigenfrequencies varying from 0.6 to 1.6 . However, it gives the ability to obtain different frequency bandgaps for waves polarized along different directions. Finally, our results show that the metamaterial under study acts as a polarizer characterized by various polarizations of the elastic waves that complete the ones obtained using fibers of circular cross sections. 
As a final remark, it is worthwhile mentioning that the localization problem that has been solved in this work and in Bonnet \& Monchiet (2017) (for cylindrical or spherical inclusions) is characterized by a null displacement at the boundary of inclusions. It provides the dynamic density in the case of large contrast, but it can also contribute for moderate contrasts to a more sophisticated elastodynamic modelling using the Craig-Bampton substructuring method (Craig \& Bampton, 1968) as in Sridhar et al. (2016).

\section{Appendix A: Computation of Mathieu functions}

\subsection{Separation constants and Fourier coefficients}

The first step is the computation of separation constants and the associated Fourier coefficients of angular Mathieu functions. Let us consider the case of Mathieu functions $c e_{2 m}$. The related separation constants and Fourier coefficients are solutions of the infinite system of linear equations (Bibby \& Peterson, 2013) characterized by a symmetric matrix:

$$
\left[\begin{array}{cccccccc}
0 & \sqrt{2} q & 0 & 0 & & \ldots & 0 & \ldots \\
\sqrt{2} q & 4 & q & 0 & & \ldots & 0 & \ldots \\
0 & q & 16 & q & & \ldots & 0 & \ldots \\
\ldots & \ldots & \ldots & \ldots & \ldots & \ldots & 0 & \ldots \\
\ldots & \ldots & \ldots & \ldots & q & (2 r)^{2} & q & \ldots \\
\ldots & \ldots & \ldots & \ldots & \ldots & \ldots & \ldots & \ldots
\end{array}\right]\left[\begin{array}{c}
\sqrt{2} A_{0} \\
A_{2} \\
A_{4} \\
\ldots \\
A_{2 r} \\
\ldots
\end{array}\right]=\Lambda\left[\begin{array}{c}
\sqrt{2} A_{0} \\
A_{2} \\
A_{4} \\
\ldots \\
A_{2 r} \\
\ldots
\end{array}\right]
$$

This relation shows that the separation constants $\Lambda$, denoted $a_{2 m}$ for the ones related to $\mathrm{ce}_{2 m}$, are the eigenvalues of the symmetrical matrix function of $q$ introduced in the lefthand side of the previous relation, while Fourier coefficients $A_{2 r}^{(2 m)}$ are obtained from the components of the related eigenvectors. The numerical computation must be effected by restricting this matrix to a finite number $N$ of lines and columns. Several codes have been built to effect this computation. We used an existing free Matlab package (Cojocaru, 2008). This package has been modified to introduce the normalization of the eigenvectors 
described in Abramowitz \& Stegun (1972), instead of the one used in the Matlab package. It is obvious that the accuracy on $a_{2 m}$ and $A_{2 r}^{(2 m)}$ increases with $N$, as seen thereafter.

\subsection{Radial Mathieu functions}

As stressed in section 3, the best convergence when computing radial Mathieu functions is achieved with the expansion of these functions along products of Bessel functions. Such an expression for $M c_{2 m}$ has been given in equation (27). Similar expressions are provided also for $M c_{2 m+1}, M s_{2 m}, M s_{2 m+1}$ in (Abramowitz \& Stegun, 1972). An important parameter is the value of $s$ in (27). As explained in section 3 , it is of prime importance to take the value of $s$ as the one (suggested by Bibby \& Peterson (2013)) maximizing $\left|A_{2 s}^{(2 m)}\right|$, more specially when dealing with high values of $q$ and small values of the radial elliptic coordinate $\xi$. This is the "tuned method" of Bibby and Peterson, already recommended by Blanch (1946) and Buren \& Boisvert (2007). Unfortunately, numerous authors are still using a fixed value of $s$ (Gutierrez-Vega, 2008; Cojocaru, 2008). It was the case for the author of the Matlab package cited previously. So, we added into this package the ability to compute the radial Mathieu functions with adaptable values of $s$ in order to use the "tuned method".

It is worthwhile noticing that the insertion of the free parameter $s$ into the expression of radial Mathieu functions appeared early (Meixner \& Schäfke, 1954; Blanch, 1946) and is mentioned in the well known Handbook of Mathematical functions (Abramowitz \& Stegun, 1972). However, it seems that its use was 330 not systematically adopted, even in recent works. Finally, a recent monograph Bibby \& Peterson (2013) shows the clear advantages to use the "tuned method".

As it can be seen from the expressions of radial Mathieu functions, their numerical values depend on the values of $A_{2 k}^{(2 m)}$. For moderate values of $q$ and $\xi$, a number of 24 values of $k$ (or $m$ ) is sufficient. However, the value of $q$ increases significantly for small aspect ratios. So, in this case and more specifically for the computation of the participation factors (with an integration starting from 
$\xi=0$ ), the computation uses up to 96 values of $m$ and $k$ (see Appendix B).

We have effected numerous verifications on the eigenfunctions in the case of the longitudinal motion (equivalent to the case of membrane vibration studied in (Troesch \& Troesch, 1973; Daymond, 1955; Wu \& Shivakumar, 2008)) and on the values of Fourier coefficients, separation constants and of the radial Mathieu functions for which numerous tabulations are available (Bibbv \& Peterson, 2013; Abramowitz \& Stegun, 1972; Bickley, 1945). More details on the convergence are given below in appendix B.

As a final remark on the computation of Mathieu functions, it can be noticed that several works were effected on the computation of asymptotic values of angular and radial Mathieu functions for large values of $q$ (Frenkel \& Portugal, 2001; Abramowitz \& Stegun, 1972). We checked that these asymptotic expressions can be used for small values of $m$ but unfortunately, they cannot be extended to the full range of values of $m$ used in the present work, even using the 5 terms expansion of Goldstein (Goldstein, 1927).

\section{Appendix B : Results on convergence}

The results depend essentially on the number of terms kept in the matrix defined in equation (36), i.e. also the number of terms of $A_{2 r}^{2 m}$. The number of values $N$ kept in the computation is the same for $r$ and $m$. The basic computation is effected with $N=24$. Next, the convergence is checked by using a second computation with $N^{\prime}=2 N=48$. The error of convergence with $N=24$ is then given by:

$$
\operatorname{Err}_{0}(N)=\left|\frac{F_{N^{\prime}}-F_{N}}{F_{N^{\prime}}}\right|,
$$

where $F$ is the quantity of interest, participation factor or resonance frequency.

From a general point of view, the accuracy on the results decreases with $q$. As a consequence, it decreases with the aspect ratio and the value of eigenfrequency. However, the main source of accuracy loss is the aspect ratio. Therefore, the accuracy is characterized at a fixed value of aspect ratio by the value of $\mathrm{Err}$ corresponding to the highest value of $\operatorname{Err}_{0}$ on the different eigenfrequencies. If 
the value of $E r r$ is higher than $10^{-4}$, the value of $N$ is multiplied by 2 . The process is iterated until convergence is reached.

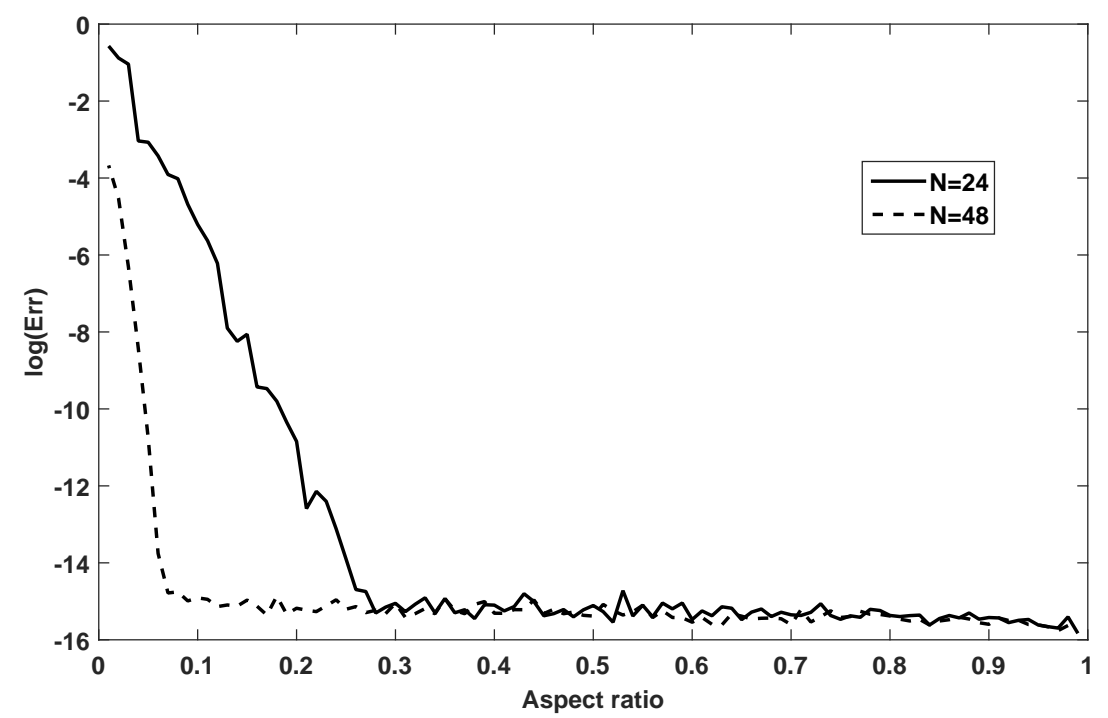

Figure 15: Error on eigenfrequencies for the longitudinal motion as a function of the aspect ratio.

\subsubsection{Longitudinal motion}

For the longitudinal motion, Fig. 15 and 16 show the value of Err for two values of $N, N=24,48$. It can be seen that a value of $N=24$ produces values of very high accuracy for aspect ratios higher than 0.2. However, for smaller aspect ratios, the value of Err increases. For both kinds of results, a value of $N=48$ leads to values of Err inferior to $10^{-4}$.

\subsubsection{Transversal motion}

The values of Err are shown in Fig. 17 and 18 for a transversal motion along $x_{1}$. It can be seen that similar results are obtained. Now, all results lead to $\operatorname{Err}<10^{-4}$ for the eigenfrequencies. On the contrary, the computation of participation factors needs to use a value of $N=96$ to produce a satisfying 


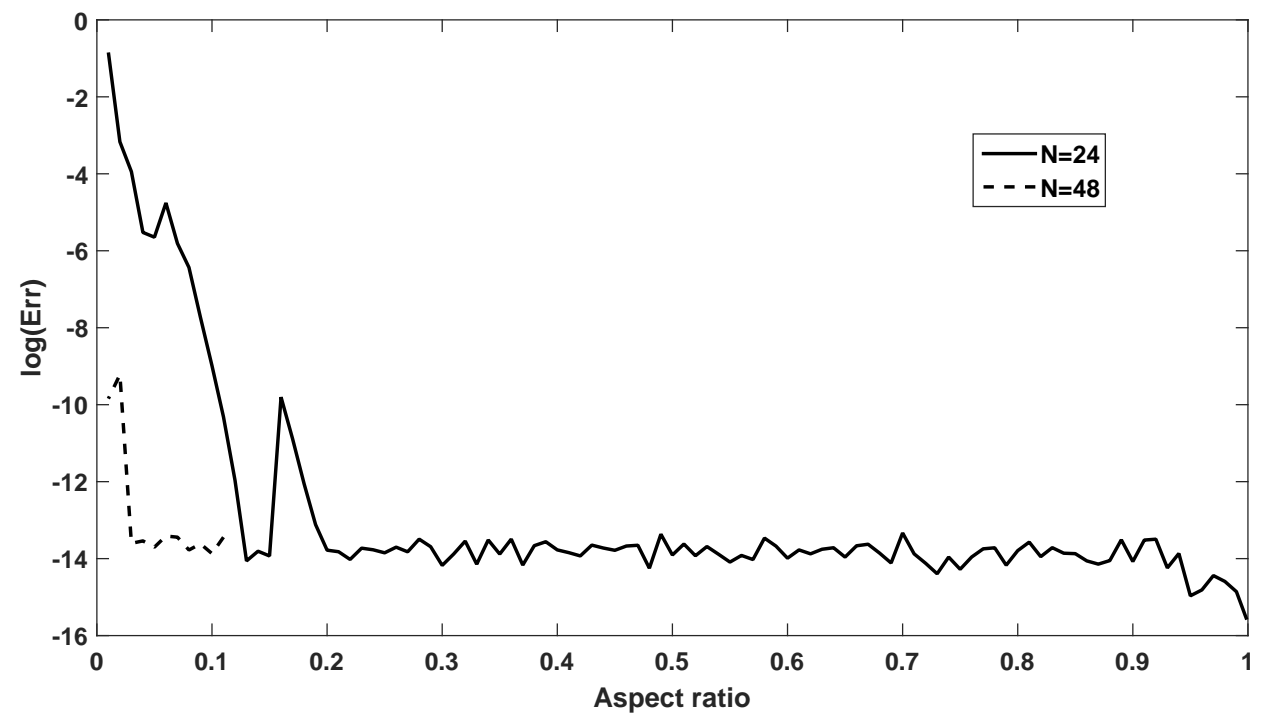

Figure 16: Error on participation factors for the longitudinal motion as a function of the aspect ratio.

value of Err. Similar results were obtained for the motion along $x_{2}$ and are not reported here. 


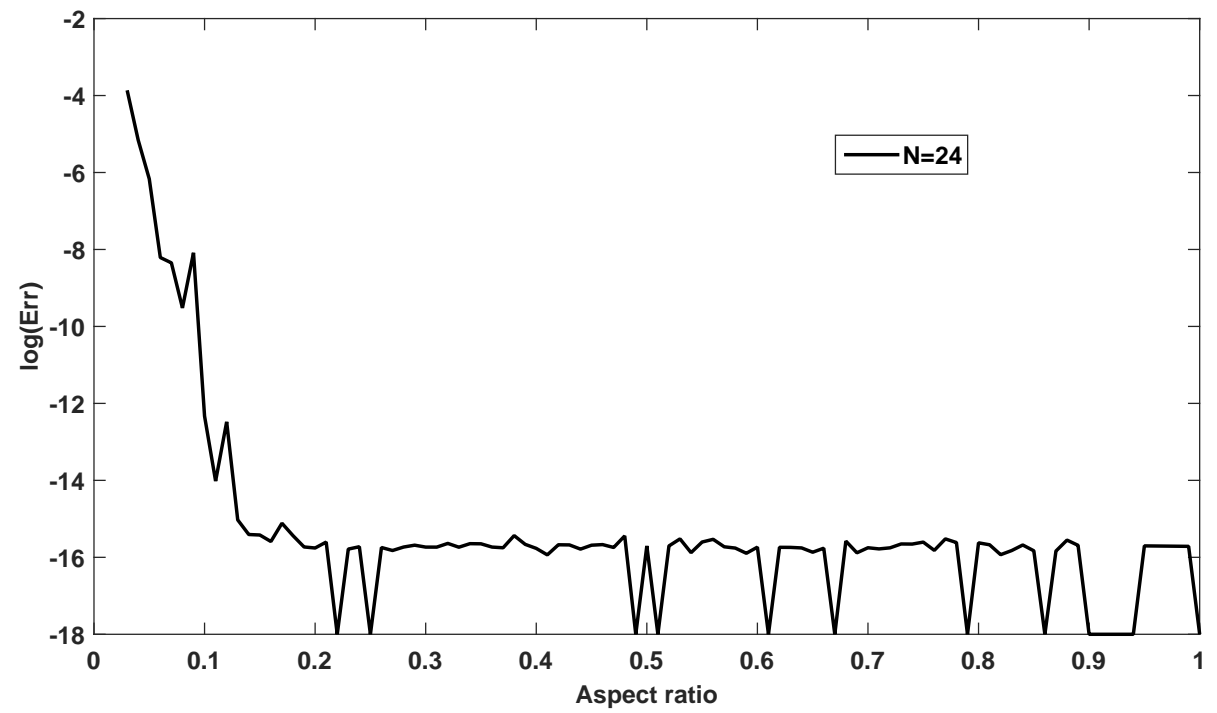

Figure 17: Error on eigenfrequencies for the transversal motion along $x_{1}$

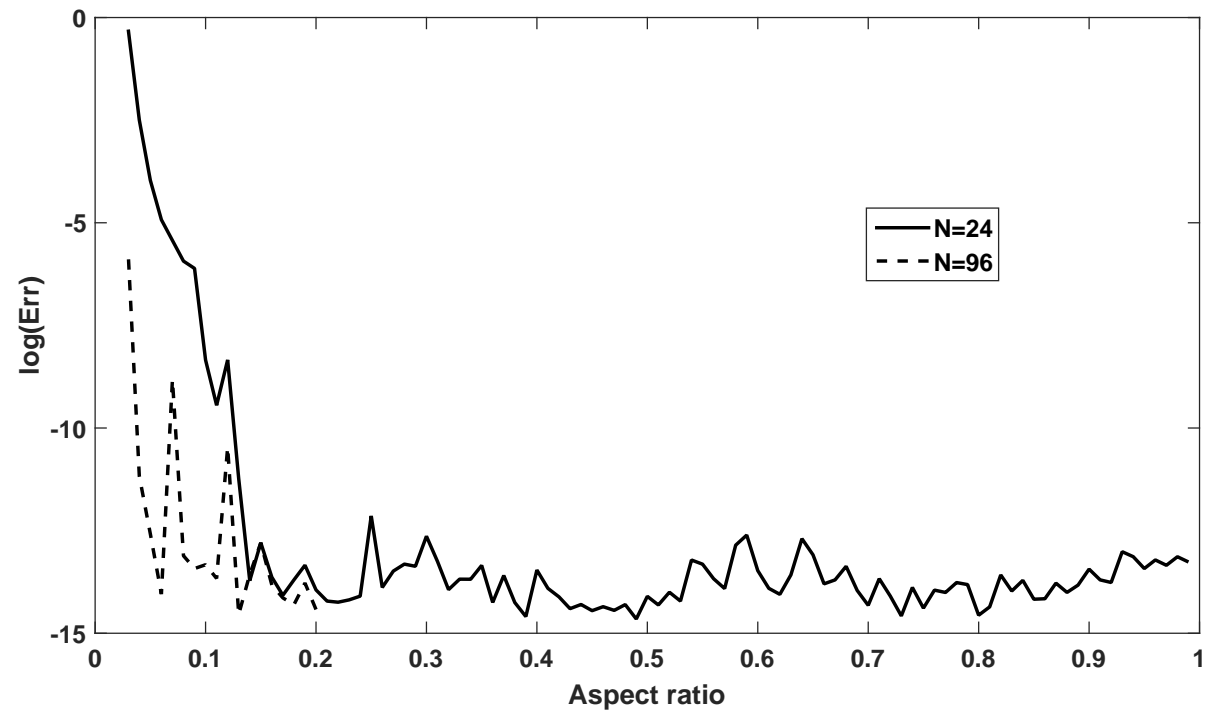

Figure 18: Error on participation factors for the transversal motion along $x_{1}$ 
11. Appendix C: Explicit expressions of integrals used in the computation of participation factors

The integrals used in the computation of $I_{3}$ for the longitudinal motion are:

$$
\begin{gathered}
T_{\eta 1(m)}=\int_{0}^{2 \pi} c e_{2 m} d \eta=2 \pi A_{0}^{(2 m)} \\
T_{\eta 2(m)}=\int_{0}^{2 \pi} c e_{2 m} \cos 2 \eta d \eta=\pi A_{2}^{(2 m)} \\
V_{\eta 1(m)}=\int_{0}^{2 \pi}\left(c e_{2 m}\right)^{2} d \eta=\pi\left[A_{0}^{(2 m) 2}+\sum_{r=0}^{\infty} A_{2 r}^{(2 m) 2}\right] \\
V_{\eta 2(m)}=\int_{0}^{2 \pi}\left(c e_{2 m}\right)^{2} \cos 2 \eta d \eta=\pi\left[\sum_{r=0}^{\infty} A_{2 r}^{(2 m)} A_{2 r+2}^{(2 m)}+A_{2}^{(2 m)} A_{0}^{(2 m)}\right]
\end{gathered}
$$

\section{References}

Abramowitz, M., \& Stegun, I. (1972). Handbook of Mathematical Functions with Formulas, Graphs, and Mathematical Tables. N.Y.: Dover.

Ancey, S., Bazzali, E., Gabrielli, P., \& Mercier, M. (2013). Elastodynamics and resonances in elliptical geometry. Journal of Physics A: General Physics, 46, 435204 .

Auriault, J. (1994). Acoustics of heterogeneous media: macroscopic behavior by homogenization. Current Topics in Acoustical Research, 1, 63-90.

Auriault, J., \& Bonnet, G. (1985). Dynamique des composites élastiques périodiques. (Dynamics of periodic elastic composites). Arch. Mech., 37, 269-284.

Auriault, J., \& Boutin, C. (2012). Long wavelength inner-resonance cut-off frequencies in elastic composite materials. International Journal of Solids and Structures, 49, 3269-3281.

790

Avila, A., Griso, G., \& Miara, B. (2005). Phononic bandgaps in linearized elasticity. Comptes Rendus de l'Académie des Sciences I, 340, 933-938. 
Babych, N., Kamotski, I., \& Smyshlyaev, V. (2008). Homogenization of spectral problems in bounded domains with doubly high contrasts. Networks and Heterogeneous Media, 3, 413-436.

Baz, A. (2010). An active acoustic metamaterial with tunable effective density. ASME Journal of Vibration and Acoustics, 132, 0410111-0410119.

Bazzali, E. (2014). Résonances d' objets élastiques en géométries elliptique et sphéroidale (Resonance of elastic objects in elliptic and spheroidal geometries). Ph.D. thesis Université de Corse France.

Bensoussan, A., Lions, J., \& Papanicolaou, G. (1978). Asymptotic analysis for periodic structures.. Amsterdam: North Holland.

Bibby, M., \& Peterson, A. (2013). Accurate computation of Mathieu functions. San Rafael, California: Morgan and Claypool.

Bickley, W. G. (1945). The Tabulation of Mathieu functions. Mathematical Tables and Other Aids to Computation, 1, 409-419.

Bigoni, D., Guenneau, S., Movchan, A. B., \& Brun, M. (2013). Elastic metamaterials with inertial locally resonant structures: Application to lensing and localization. Physical Review B, 84, 174303.

Blanch, G. (1946). On the computation of Mathieu functions. Journal of Mathematics and Physics, 25, 1-20.

Bonnet, G., \& Monchiet, V. (2017). Dynamic mass density of resonant metamaterials with homogeneous inclusions. Journal of the Acoustical Society of America, 142, 890-901.

Boutin, C., Auriault, J., \& Bonnet, G. (2018). Inner resonance in media gov815 erned by hyperbolic and parabolic dynamic equations. Principle and examples. In H. Altenbach, J. Pouget, M. Rousseau, B. Collet, \& T. Michelitsch (Eds.), Generalized Models and Non-classical Approaches in Complex Materials. chapter 6. (pp. 83-84). Berlin: Springer. 
Buren, A. V., \& Boisvert, J. (2007). Accurate calculation of the modified Mathieu functions of integer order. Quarterly of Applied Mathematics, 65, 1-23.

Cojocaru, E. (2008). Mathieu functions, computational toolbox implemented in matlab. Https://arxiv.org/pdf/0811.1970.pdf.

Comi, C., \& Marigo, J. (2020). Homogenization approach and Bloch-Floquet theory for band-gap prediction in 2D locally resonant metamaterials. Journal of Elasticity, 139, 61-90.

Craig, R., \& Bampton, M. (1968). Coupling of substructures for dynamic analyses. AIAA Journal, 6, 1313-1319.

Craster, R., Kaplunov, J., \& Pichugin, A. (2010). High-frequency homogenization for periodic media. Proceedings of the Royal Society A, 466, 2341-2362.

Daymond, S. (1955). The principal frequencies of vibrating systems with elliptic boundaries. Quarterly Journal of Mechanics and Applied Mathematics, 8, $361-372$.

Eringen, A., \& Suhubi, E. (1978). Elastodynamics, Vol.2.. N.Y.: Acad. Press.

Frenkel, D., \& Portugal, R. (2001). Algebraic methods to compute Mathieu functions. Journal of Physics A: General Physics, 34, 3541-3551.

Goldstein, S. (1927). Mathieu functions. Transactions of the Cambridge Philosophical Society, 23, 303-336.

Gutierrez-Vega, J. C. (2008). Theory and numerical analysis of the Mathieu functions. Ph.D. thesis Instituto Nacional de Astrofisica, Optica y Electronica Puebla, Mexico.

Hackman, R. (1984). The transition matrix for acoustic and elastic wave scattering in prolate spheroidal coordinates. Journal of the Acoustical Society of America, 75, 35-45. 
Huang, H., \& Sun, C. (2011). Locally resonant acoustic metamaterials with 2D anisotropic effective mass density. Philosophical Magazine, 91, 981-996.

Huang, H., \& Sun, C. (2012). Anomalous wave propagation in a one-dimensional acoustic metamaterial having simultaneously negative mass density and Young's modulus. Journal of the Acoustical Society of America, 132, 28872895.

Krokhin, A., Arriaga, J., \& Gumen, L. (2003). Speed of sound in periodic elastic composites. Physical Review Letters, 91, 2643021-2643024.

Lee, S., Park, C., Seo, Y., Wang, Z., \& Kim, C. (2009). Acoustic metamaterial with negative density. Physical letters A, 373, 4464-4469.

Liu, Z., Zhang, X., Mao, Y., Zhu, Y., Yang, Z., Chan, C., \& Sheng, P. (2000). Locally resonant materials. Science, 289, 1734-1736.

Mathieu, E. (1868). Le mouvement vibratoire d'une membrane de forme elliptique (Vibration of an elliptic membrane). Journal de Mathématiques Pures et Appliquées, 13, 137-203.

Meixner, J., \& Schäfke, F. (1954). Mathieusche Funktionen and Spheroidfunktionen. Berlin: Springer Verlag.

Milton, G. (2002). The theory of composites. Cambridge: Cambridge Univ. Press.

Milton, G. (2007). New metamaterials with macroscopic behavior outside that of continuum elastodynamics. New Journal of Physics, 9, 359.

Milton, G., \& Willis, J. (2007). On modifications of Newton's second law and linear continuum elastodynamics. Proceedings of the Royal Society A, 463, $855-880$.

Morse, P., \& Feshbach, H. (1953). Methods of theoretical physics. N.Y.: McGrawHill. 
870 Naify, C., Chang, C., Mcknight, G., \& Nutt, S. (2012). Scaling of membranetype locally resonant acoustic metamaterial arrays. Journal of the Acoustical Society of America, 132, 2784-2792.

Nassar, H., He, Q., \& Auffray, N. (2015). Willis elastodynamic homogenization theory revisited for periodic media. Journal of the Mechanics and Physics of Solids, r7, 158-178.

Nassar, H., He, Q., \& Auffray, N. (2016). On asymptotic elastodynamic homogenization approaches for periodic media. Journal of the Mechanics and Physics of Solids, 88, 274-290.

Nemat-Nasser, S., Willis, J., Srivastava, A., \& Amirkhizi, A. (2011). Homogenization of periodic elastic composites and locally resonant materials. Physical Review B, 83, 104103.

Norris, A. N., Shuvalov, A. L., \& Kutsenlo, A. A. (2012). Analytical formulation of three-dimensional dynamic homogenization for periodic elastic systems. Proceedings of the Royal Society A, 468, 1629-1651.

${ }_{885}$ Park, J., Park, B., Kim, D., \& Park, J. (2012). Determination of effective mass density and modulus for resonant metamaterials. Journal of the Acoustical Society of America, 132, 2793-2799.

Parnell, W., \& Abrahams, I. (2006). Dynamic homogenization in periodic fibre reinforced media. Quasi-static limit for $\mathrm{SH}$ waves. Wave Motion, 43, 474-498.

Rallu, A., Hans, S., \& Boutin, C. (2018). Asymptotic analysis of high-frequency modulation in periodic systems. Analytical study of discrete and continuous structures. Journal of the Mechanics and Physics of Solids, 117, 123-156.

Shamonina, E., \& Solymar, L. (2007). Metamaterials: How the subject started. Metamaterials, 1, 12-18.

895 Sheng, P., Zhang, X., Liu, Z., \& Chan, C. (2003). Locally resonant sonic materials. Physica B, 338, 201-205. 
Soubestre, J., \& Boutin, C. (2012). Non-local dynamic behavior of linear fiber reinforced materials. Mechanics of materials, 55, 16-32.

Sridhar, A., Kouznetsova, V., \& Geers, M. (2016). Homogenization of locally resonant acoustic metamaterials towards an emergent enriched continuum. Computational Mechanics, 57, 423-435.

Sridhar, A., Kouznetsova, V., \& Geers, M. (2018). A general multiscale framework for the emergent effective elastodynamics of metamaterials. Journal of the Mechanics and Physics of Solids, 111, 414-433.

${ }_{905}$ Troesch, B. A., \& Troesch, H. R. (1973). Eigenfrequencies of an elliptic membrane. Mathematics of Computation, 27, 16-32.

Vasseur, J., Deymier, P., Prantzikonis, G., \& Hong, G. (1998). Experimental evidence for the existence of absolute acoustic band gaps in two-dimensional periodic composite media. Journal of Physics: Condensed matter, 10, 60516064 .

Veselago, V. (1968). The electrodynamics of substances with simultaneously negative values of $\epsilon$ and $\mu$. Soviet Physics Uspekhi, 10, 509-514.

Willis, J. (1981). Variational principles for dynamic problems for inhomogeneous elastic media. Wave Motion, 3, 1-11.

Willis, J. (2009). Exact effective relations of s laminated body. Mechanics of materials, 41, 385-393.

Wu, P., \& Shivakumar, P. (2008). Eigenvalues of the laplacian on a elliptic domain. Computers and Mathematics with Applications, 55, 1129-1136.

Yang, Z., Mei, J., Yang, M., Zhou, N. C., \& Sheng, P. (2008). Membrane-type 920 acoustic metamaterial with negative dynamic mass. Physical Review Letters, $101,204301$.

Yao, S., Zhou, X., \& Hu, G. (2008). Experimental study on negative effective mass in a 1D mass-spring system. New Journal of Physics, 10, 043020. 
Zhou, X., Liu, X., \& Huc, G. (2012). Elastic metamaterials with local resonances: an overview. Theoretical and applied mechanics letters, 2, 041001. 